\title{
On the Distortion SNR Exponent of Some Layered Transmission Schemes
}

\author{
Kapil Bhattad*, Krishna Narayanan *, and Giuseppe Caire ${ }^{\dagger}$ \\ *Texas A \& M University, College Station, TX 77843. kbhattad,krn@ece.tamu.edu \\ $\dagger$ University of Southern California, Los Angeles, CA 90089. caire@usc.edu
}

\begin{abstract}
We consider the problem of joint source-channel coding for transmitting $K$ samples of a complex Gaussian source over $T=b K$ uses of a block-fading multiple input multiple output (MIMO) channel with $M$ transmit and $N$ receive antennas. We consider the case when we are allowed to code over $L$ blocks. The channel gain is assumed to be constant over a block and channel gains for different blocks are assumed to be independent. The performance measure of interest is the rate of decay of the expected mean squared error with the signal-to-noise ratio (SNR), called the distortion SNR exponent. We first show that using a broadcast strategy as in [1], but with a different power and rate allocation policy, the optimal distortion SNR exponent can be achieved for bandwidth efficiencies $0 \leq b<(|N-M|+1) / \min (M, N)$. This is the first time the optimal exponent is characterized for $1 / \min (M, N)<b<(|N-M|+1) / \min (M, N)$. Also, for $b>M N L^{2}$, we show that the broadcast scheme achieves the optimal exponent of $M N L$. Special cases of this result have been derived in [1] for the $L=1$ case and in [2] for $M=N=1$. We then propose a digital layered transmission scheme that uses both time layering and superposition. This includes many known schemes in [1,3] as special cases. The proposed scheme is at least as good as the currently best known schemes for the entire range of bandwidth efficiencies, whereas at least for some $M, N$, and $b$, it is strictly better than the currently best known schemes.
\end{abstract}

\section{INTRODUCTION}

\section{A. Problem Statement}

Consider the problem of transmitting $K$ samples of a complex Gaussian source in $T=b K$ uses of an $M \times N$ MIMO channel with block fading where $b$ is the ratio of the channel bandwidth to the source bandwidth. The channel is given by

$$
\mathbf{y}_{t}=\sqrt{\frac{\rho}{M}} \mathbf{H}_{\left\lceil\frac{L t}{T}\right\rceil} \mathbf{x}_{t}+\mathbf{w}_{t}, \quad t=1, \ldots, T
$$

where: $T$ is the duration (in channel uses) of the transmitted block; $\mathbf{H}_{l} \in \mathbb{C}^{N \times M}, l=1, \ldots, L$, is the channel matrix for $\frac{(l-1) T}{L}<t \leq \frac{l T}{L}$ containing random i.i.d. elements $h_{i, j}^{l} \sim \mathcal{C N}(0,1)$ (Rayleigh independent fading). The channel matrix for different blocks are independent; $\mathbf{x}_{t}$ is the transmitted signal at time $t$; the transmitted codeword, $\mathbf{X}=\left[\mathbf{x}_{1}, \ldots, \mathbf{x}_{T}\right]$, is normalized such that $\operatorname{tr}\left(\mathbb{E}\left[\mathbf{X}^{\mathrm{H}} \mathbf{X}\right]\right) \leq M T$; $\mathbf{w}_{t} \sim \mathcal{C N}\left(0, I^{M \times M}\right)$ is additive white Gaussian noise; $\rho$ denotes the Signal-to-Noise Ratio (SNR), defined as the ratio of the average received signal energy per receiving antenna to the noise per-component variance. We also define $m=\min \{M, N\}$ and $n=\max \{M, N\}$. 
When the channel state information is available at both the transmitter and the receiver, Shannon's separation theorem applies and separate source and channel coding is optimal. However, when the channel state information is available only at the receiver, the separation theorem fails and the optimal scheme requires joint source and channel coding.

Consider a family of joint source-channel coding schemes $\left\{\mathcal{S C}_{b}(\rho)\right\}$ of spectral efficiency $1 / b$, where $\mathcal{S C}_{b}(\rho)$ denotes the scheme that operates at $\operatorname{SNR} \rho$. Corresponding to the coding scheme $\mathcal{S C}_{b}(\rho)$, let $D(\rho)$ denote the distortion averaged over the source, the noise, and the channel realization. The distortion SNR exponent of the family is defined as the limit

$$
a(b)=-\lim _{\rho \rightarrow \infty} \frac{\log D(\rho)}{\log \rho} .
$$

The distortion SNR exponent of the channel, denoted by $a^{\star}(b)$, is the supremum of $a(b)$ over all possible coding families. We are interested in characterizing $a^{\star}(b)$ for the block fading MIMO channel.

\section{B. Prior Work}

The diversity multiplexing tradeoff [4] is closely related to the problem considered here. In [4], Zheng and Tse consider the problem of transmitting digital information over a MIMO fading channel. For a family of coding schemes $C_{r}(\rho)$ whose rate grows as $r \log \rho$, where $r$ is referred to as the multiplexing rate, the diversity order of the family is defined as the limit

$$
d(r)=-\lim _{\rho \rightarrow \infty} \frac{\log P_{e}(\rho)}{\log \rho}
$$

where $P_{e}(\rho)$ denotes the probability of decoding error corresponding to the coding scheme $C(\rho)$. The diversity order of the channel, $d^{*}(r)$, is the supremum of $d(r)$ taken over all possible coding families. In [4], for the Rayleigh fading channel, the diversity order was determined to be

$$
d^{*}(r)=(M-k)(N-k)-(M+N-1-2 k)(r-k)
$$

where $k=\lfloor r\rfloor$ for $0<r<m$ and 0 for $r>m$.

The distortion SNR exponent problem has been considered previously by many researchers in [1-3, 5-13]. Distortion SNR exponent was first defined by Laneman et al. in [11]. In [11-13] the authors compared the performance of two schemes for parallel fading channels (a) a separation based scheme and (b) a multiple description based scheme where the message sent on each channel corresponded to a description. If the multiplexing rate of the channel code is low the probability of outage is low. However, the corresponding quantization error is large. When the multiplexing rate is increased quantization error decreases but outage probability increases. For these schemes, the optimal multiplexing rate is chosen such that it maximizes the distortion SNR exponent. Goldsmith and Holliday $[9,10]$ consider a separation based scheme for the MIMO channel and derive the optimal operating point (multiplexing rate of the channel code) that maximizes the distortion SNR exponent.

An upper bound on $a^{\star}(b)$ was derived by Caire and Narayanan [5-7] and by Gunduz and Erkip [1-3, 8] by assuming that the transmitter is informed of the channel realization $\mathbf{H}=\left\{\mathbf{H}_{1}, \ldots, \mathbf{H}_{L}\right\}$. In this 
case, Shannon's separation theorem applies and the optimal distortion is given by $D(\mathbf{H})=2^{-2 R(\mathbf{H})}$ where $R(\mathbf{H})=\frac{1}{L} \sum_{l} \log \operatorname{det}\left(I+\frac{\rho}{M} \mathbf{H}_{l} \mathbf{H}_{l}^{H}\right)$. The distortion SNR exponent is then the exponent corresponding to $E_{\mathbf{H}}[D(\mathbf{H})]$. This has been computed in closed form for the Rayleigh fading channel in [1-3,5-8] and is given by

$$
a_{I T}(b)=\sum_{i=1}^{m} \min (b,(2 i-1+n-m) L) .
$$

Note that this is an upper bound and is not known to be achievable.

The schemes by Laneman et al. [11-13] and Goldsmith and Holliday $[9,10]$ are far away from the informed transmitter upper bound. In [5-7], two hybrid digital analog (HDA) scheme were proposed for $b<1 / m$ and $b>1 / m$. For $b<1 / m$, in the HDA scheme, the transmitted signal was chosen to be a superposition of an analog layer with a digital layer. The analog layer is formed by a fraction $m b$ of the source symbols. The remaining source symbols were quantized and transmitted in the digital layer. The scheme was shown to achieve the upper bound for $b<1 / m$. For $b>1 / m$, the HDA scheme involved transmitting in two "time" layers (i.e., two layers multiplexed in time). A digital layer of bandwidth $b-1 / m$ ( $T-K / m$ channel uses) was used to transmit the quantized source and the quantization error was transmitted in an analog layer of bandwidth $1 / \mathrm{m}$. This scheme improved on the exponent obtained by the separation based scheme. However, the gap to the upper bound was still large.

In $[1,3,8]$, Gunduz and Erkip proposed a hybrid layering scheme (HLS) that improved on the exponent obtained by the HDA scheme for $b>1 / m$ by allowing for multiple digital time layers instead of the single digital layer of the HDA scheme. They also proposed a broadcast scheme (BS) that involved transmitting a superposition of several digital layers. For the $L=1$ case, the broadcast scheme was shown to achieve an exponent of $M N$ for $b>M N$ which overlaps with the upper bound and is hence optimal. In this case, for the region $1 / m<b<M N$, a characterization of the best achievable distortion SNR exponent is not available. Currently the best known exponents are obtained by the hybrid layering scheme and broadcast strategy of Gunduz and Erkip [1]. In [2], Gunduz and Erkip considered the broadcast scheme for parallel channels which corresponds to $M=N=1$ and $L>1$ in our model and they showed that the broadcast scheme achieves an exponent of $M N L$ for $b>L^{2}$ and is hence optimal. Note that throughout this paper we refer to a superposition coding scheme as a broadcast scheme.

In other related work, Dunn and Laneman [14] consider the distortion to be of the form

$$
D \approx C(b) \log (b \rho)^{p} \rho^{-a(b)}
$$

and compare several schemes using this approximation.

\section{Main Results}

The main results presented in this paper are summarized below.

1) We fully characterize the exponent achievable by any broadcast (superposition) scheme. An achievable exponent and the corresponding rate and power allocation are specified in Theorem 2.1. In Theorems 2.2 and 2.3, we show that no broadcast scheme can outperform the scheme in Theorem 2.1 
2) We show that the broadcast scheme in [1] when used with a different power and rate allocation than that specified in [1] achieves the optimal exponent $m b$ for $b<\frac{n-m+1}{m}$.

3) The broadcast scheme with the proposed power and rate allocation policy achieves the optimal exponent of $M N L$ for $b>M N L^{2}$. Special cases of this result have been derived in [1] for the $L=1$ case and in [2] for $M=N=1$.

4) The proposed power and rate allocation policy for the broadcast scheme becomes identical to that specified in [1] for $M N L-(M+N-1) L<b<M N L-(M+N-1)(L-1)$. For other $b<M N L^{2}$ the distortion SNR exponent obtained is larger than the broadcast scheme exponent of [1].

5) We propose a time layering scheme in which the last time layer is a broadcast layer, i.e, the last time layer is a superposition of several layers. The distortion SNR exponent obtained using this scheme is shown to be better than the exponent obtained using the HLS scheme of [1]. We refer to this scheme as LSBLEND as an abbreviation for Layered Scheme with a Broadcast Layer at the end.

6) We also propose a layering strategy, termed the Box scheme, which generalizes BS and LSBLEND proposed in this paper and the strategies considered earlier in [1,5] by allowing for superposition and time layers simultaneously. All previously known schemes are special cases of the Box scheme and hence the Box scheme performs at least as well as these schemes. However, the optimal distortion SNR exponent for the Box scheme is difficult to obtain. We present a suboptimal algorithm to compute an achievable distortion SNR exponent. The scheme with the suboptimal algorithm is shown to outperform all previously known schemes, including BS and LSBLEND which are proposed in this paper, for some range of $b$, whereas, for the considered examples, they are at least as good as previously known schemes for all $b$.

Some of these results have been reported in a conference version of this paper [15].

\section{Organization of the paper}

The paper is organized as follows. The proposed schemes - Broadcast Scheme, LSBLEND, and Box

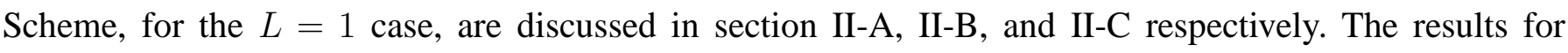
$L>1$ case are presented in section III. In section IV] we present some examples that demonstrate that the proposed schemes achieve better distortion SNR exponent than previously known schemes and finally we conclude in section $\mathrm{V}$.

\section{Proposed Schemes}

In this section we present the proposed schemes for the $\mathrm{L}=1$ case. The results for the $L>1$ case are presented in section $\amalg$.

\section{A. Digital Layering using Superposition Only}

Consider the broadcast scheme shown in Fig. 1. The scheme has $N_{s}$ superposition layers with the $i$ th superposition layer being assigned a power level of $\rho^{\gamma_{i-1}}-\rho^{\gamma_{i}}$ where $\rho$ is the signal-to-noise ratio and 


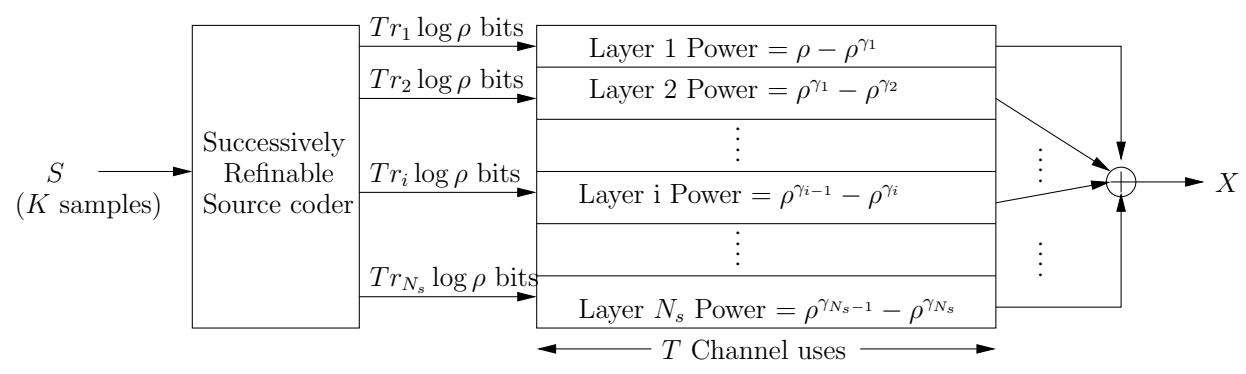

Fig. 1. Broadcast Schemes

$\gamma_{i} \geq 0$ is a decreasing sequence with $\gamma_{0}=1$. The source is compressed into $N_{s}$ layers such that it is successively refinable. The rate in the $i$ th refinement layer is $\frac{T r_{i} \log \rho}{K}=b r_{i} \log \rho$. Therefore, if a receiver estimates the source using the first $J$ layers the resulting distortion would be $2^{-\sum_{i=1}^{J} b r_{i} \log \rho}=\rho^{-b \sum_{i=1}^{J} r_{i}}$. The $i$ th refinement layer is transmitted in the $i$ th superposition layer. Since $\operatorname{Tr}_{i} \log \rho$ bits have to be transmitted in $T$ uses of the channel, the resulting rate of transmission in the $i$ th broadcast layer is $r_{i} \log \rho$. For mathematical convenience in deriving the expressions, we will assume that in the last layer (layer $N_{s}+1$ ) the remaining power of $\rho^{\gamma_{N_{s}}}$ is used to transmit Gaussian noise. Therefore, $\gamma_{N_{s}+1}=0$ and $r_{N_{s}+1}=0$. The channel codes used in the broadcast layers are assumed to achieve the diversity multiplexing tradeoff [4] corresponding to that layer. Here achieving the diversity multiplexing tradeoff refers to achieving an error probability that decays as $\rho^{-d(r)}$ with a coding rate that grows as $r \log \rho$, where $d(r)$ is the optimal diversity multiplexing tradeoff function corresponding to that layer.

At the receiver, the decoder attempts to decode as many layers as it can using successive interference cancellation starting from the first layer. That is, it decodes layer 1 by treating the signal transmitted in layers 2 to $N_{s}$ as noise. On successful decoding it removes the contribution of layer 1 from the received signal and repeats the process for layer 2 and so on. It then makes an estimate of the source using all the layers it is able to decode.

To compute the distortion SNR exponent of the broadcast scheme, we first characterize the diversity multiplexing tradeoff of the broadcast scheme in the following lemma.

Lemma 2.1: If the multiplexing gain in the $i$ th layer of the broadcast scheme is $r_{i}=k\left(\gamma_{i-1}-\gamma_{i}\right)+\delta$ where $k \in\{0,1, \ldots, m-1\}$ and $0 \leq \delta<\gamma_{i-1}-\gamma_{i}, \gamma_{i-1}>\gamma_{i} \geq 0$, then, the achievable diversity in the $i$ th layer of the broadcast scheme, assuming that the message transmitted in the previous layers is available at the receiver, is given by

$$
d^{\star}\left(r_{i}, \gamma_{i-1}, \gamma_{i}\right)=(m-k)(n-k) \gamma_{i-1}-(m+n-1-2 k) \delta .
$$

That is, if

$$
X=\frac{1}{\sqrt{\rho}}\left(\sum_{i=1}^{N_{s}} \sqrt{\left(\rho^{\gamma_{i-1}}-\rho^{\gamma_{i}}\right)} X_{i}+\sqrt{\rho^{\gamma_{N_{s}}}} N_{1}\right),
$$

where $X_{i}, N_{1} \sim \mathcal{C N}\left(0, I^{M \times M}\right)$, is transmitted over a MIMO channel $Y=\sqrt{\frac{\rho}{M}} \mathbf{H} X+N$, then the probability of the outage event

$$
\mathcal{A}_{i}=\left\{H: I\left(X_{i} ; Y \mid \mathbf{H}=H, X_{1}, \ldots X_{i-1}\right)<r_{i} \log \rho\right\}
$$


is given by $P\left(\mathcal{A}_{i}\right) \doteq \rho^{-d^{\star}\left(r_{i}, \gamma_{i-1}, \gamma_{i}\right)}$. (Here $A \doteq B$ is used to denote that $A$ and $B$ are equal in exponential order, i.e., $\lim _{\rho \rightarrow \infty} \frac{\log A}{\log \rho}=\lim _{\rho \rightarrow \infty} \frac{\log B}{\log \rho}$.) Note that the term $\sqrt{\rho^{\gamma N_{s}}} N_{1}$ in $X$ is the Gaussian noise transmitted in layer $N_{s}+1$ and is introduced for mathematical convenience. It should not be confused with noise from the channel.

Proof:

$$
P\left(\mathcal{A}_{i}\right)=P\left(\log \frac{\operatorname{det}\left(I+\frac{1}{M} \rho^{\gamma_{i-1}} \mathbf{H H}^{H}\right)}{\operatorname{det}\left(I+\frac{1}{M} \rho^{\gamma_{i}} \mathbf{H H}^{H}\right)}<r_{i} \log \rho\right) .
$$

Let $\lambda_{1}, \ldots, \lambda_{m}$ denote the non-zero ordered eigenvalues of $\mathbf{H H}^{H}$ with $\lambda_{1} \leq \lambda_{2} \leq \ldots \lambda_{m}$. As in [4], let $\alpha_{j}=-\frac{\log \lambda_{j}}{\log \rho}$. Therefore, $\alpha_{1} \geq \alpha_{2} \geq \ldots \alpha_{m}$. Then

$$
P\left(\mathcal{A}_{i}\right)=P\left(\log \prod_{j=1}^{m} \frac{1+\frac{1}{M} \rho^{\gamma_{i-1}-\alpha_{j}}}{1+\frac{1}{M} \rho^{\gamma_{i}-\alpha_{j}}}<r_{i} \log \rho\right) .
$$

At high SNR, we obtain $P\left(\mathcal{A}_{i}\right) \doteq P\left(\mathcal{A}^{\prime}\right)$ where

$$
\mathcal{A}^{\prime}=\left\{\alpha: \sum_{j=1}^{m}\left(\gamma_{i-1}-\alpha_{j}\right)^{+}-\sum_{j=1}^{m}\left(\gamma_{i}-\alpha_{j}\right)^{+}<r_{i}\right\} .
$$

Starting from Lemma 3 of [4] and following in the footsteps of [4] we obtain

$$
P\left(\mathcal{A}^{\prime}\right)=\int_{\mathcal{A}^{\prime}} p(\alpha) d \alpha \doteq \int_{\mathcal{A}^{\prime} \cap \boldsymbol{\alpha}^{+}} \prod_{j=1}^{m} \rho^{(2 j-1+n-m) \alpha_{j}} d \alpha
$$

for the Rayleigh fading channel. Therefore the outage probability is given by $P\left(\mathcal{A}_{i}\right) \doteq \rho^{-d^{\star}\left(r_{i}, \gamma_{i-1}, \gamma_{i}\right)}$ where

$$
d^{\star}\left(r_{i}, \gamma_{i-1}, \gamma_{i}\right)=\inf _{\mathcal{A}^{\prime} \cap \boldsymbol{\alpha}^{+}} \sum_{j=1}^{m}(2 j-1+n-m) \alpha_{j} .
$$

For $r_{i}=k\left(\gamma_{i-1}-\gamma_{i}\right)+\delta$ where $k \in[0,1, \ldots, m-1]$ and $0 \leq \delta<\gamma_{i-1}-\gamma_{i}$, the infimum in (14) occurs when $\alpha=\alpha^{*}$ where

$$
\alpha_{j}^{*}= \begin{cases}\gamma_{i-1}, & 1 \leq j<m-k \\ \gamma_{i-1}-\delta, & j=m-k \\ 0, & m-k<j \leq m .\end{cases}
$$

Substituting $\alpha^{*}$ in (14) we obtain

$$
\begin{aligned}
d^{\star}\left(r_{i}, \gamma_{i-1}, \gamma_{i}\right) & =\left(\sum_{j=1}^{m-k-1}(2 j-1+n-m)\right) \gamma_{i-1}+(2(m-k)-1+n-m)\left(\gamma_{i-1}-\delta\right) \\
& =\left(\sum_{j=1}^{m-k}(2 j-1+n-m)\right) \gamma_{i-1}-(2(m-k)-1+n-m) \delta \\
& =(m-k)\left(2 \frac{m-k+1}{2}-1+n-m\right) \gamma_{i-1}-(m+n-1-2 k) \delta .
\end{aligned}
$$

This then gives the desired result.

Note that the probability of the outage event $\mathcal{A}_{i}$ discussed in lemma 2.1 is different from (a) the outage probability of layer $i$ and (b) the outage probability of layer $i$ given layers 1 to $i-1$ are decoded. Rather, 


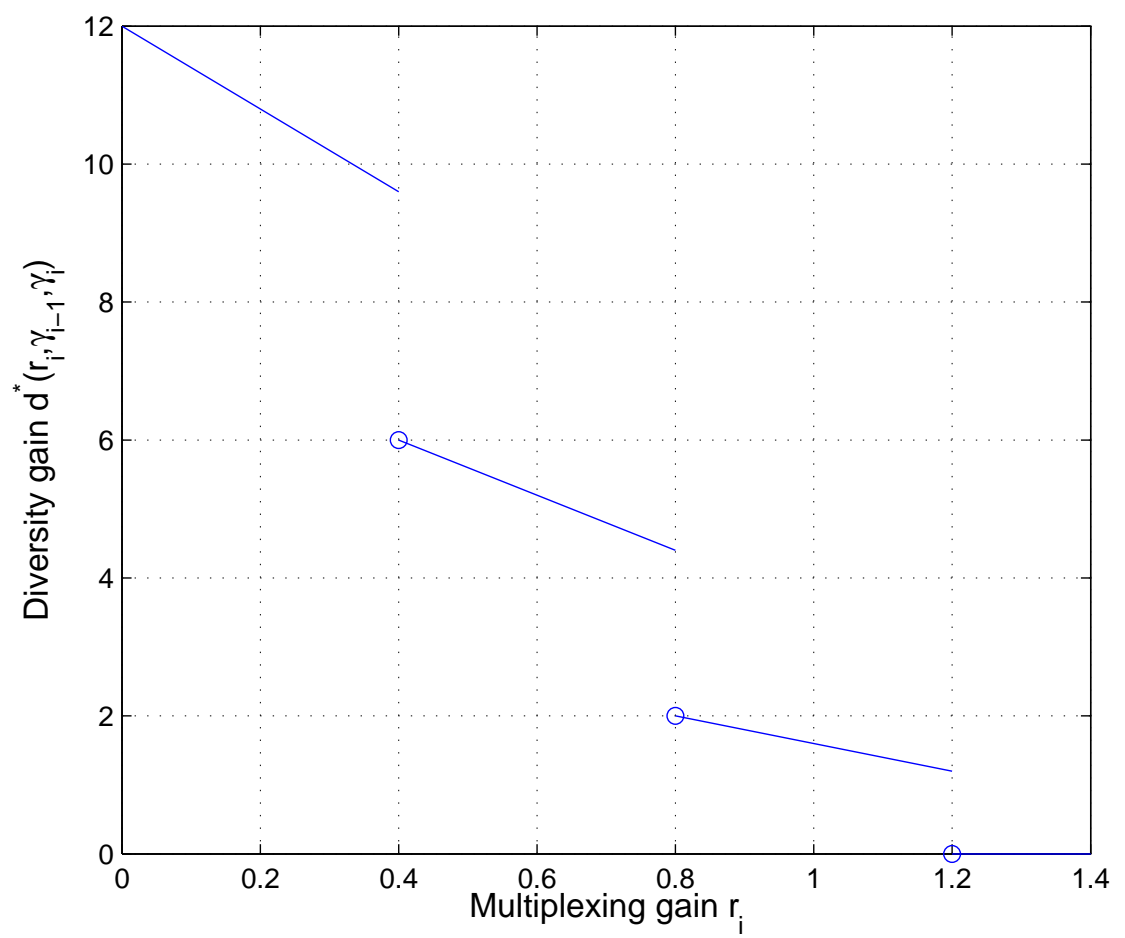

Fig. 2. Diversity Multiplexing tradeoff corresponding to a broadcast layer with $\gamma_{i-1}=1$ and $\gamma_{i}=0.6$ for a $3 \times 4$ MIMO system

it is the probability of outage of layer $i$ with a genie aided decoder where the genie provides the signal that is transmitted in layers 1 to $i-1$.

We will refer to the rate of decay of $P\left(\mathcal{A}_{i}\right)$ with $\rho$, i.e., $\lim _{\rho \rightarrow \infty} \frac{\log P\left(\mathcal{A}_{i}\right)}{\log \rho}=d^{*}\left(r_{i}, \gamma_{i-1}, \gamma_{i}\right)$, as the diversity of layer $i$. In Fig. 2, as an example, the diversity multiplexing tradeoff corresponding to a superposition layer in the broadcast scheme is plotted. Note that it is discontinuous.

Note that the diversity multiplexing tradeoff of Zheng and Tse [4] specified in (4) corresponds to the case when $\gamma_{i-1}=1$ and $\gamma_{i}=0$. From Lemma 2.1 and (4) we can verify that $d^{*}\left(r_{i}, 1,0\right)=d^{*}\left(r_{i}\right)$. To keep the notation brief, in such cases, we will use $d^{*}\left(r_{i}\right)$ in place of $d^{*}\left(r_{i}, 1,0\right)$.

The broadcast scheme considered in [1] used $r_{i}=\gamma_{i-1}-\gamma_{i}$ and optimized the power allocation, $\gamma_{i}$ 's, in order to maximize the distortion SNR exponent. With this rate and power allocation, the resulting scheme had a distortion SNR exponent equal to $\min (b, M N)$. We show that by using a different rate and power allocation than that specified in [1], we can obtain the optimal exponent of $m b$ for any $b<\frac{n-m+1}{m}$. Notice that in this region the broadcast scheme with the rate and power allocation specified in [1] performs quite poorly. Our main result in this section is the following theorem.

Theorem 2.1: The broadcast scheme achieves a distortion SNR exponent of $(k+1) b, k \in\{0,1, \ldots, m-$ 1) for $\frac{(M-k-1)(N-k-1)}{k+1}<b<\frac{(M-k)(N-k)}{k+1}$ with power and rate allocation

$$
\gamma_{i}=\left(\frac{b(k+1)-(M-k-1)(N-k-1)}{(M-k)(N-k)-(M-k-1)(N-k-1)}\right)^{i}
$$


and

$$
r_{i}=(k+1)\left(\gamma_{i-1}-\gamma_{i}\right)-\epsilon
$$

for arbitrarily small $\epsilon>0$.

Proof: The distortion is given by

$$
D=\sum_{i=1}^{N_{s}} P(\text { Layer } 1 \text { to } i-1 \text { decoded, layer } i \text { decoding failed }) D_{i}+P(\text { All layers decoded }) D_{N_{s}+1}
$$

where $D_{i}$ is the distortion when only the first $i-1$ layers are used for reconstructing the source. If the layers $1, \ldots, i-1$ can be decoded, a source coding rate of $b \sum_{j=1}^{i-1} r_{j}$ can be obtained. Therefore $D_{i}=\rho^{-b \sum_{j=1}^{i-1} r_{j}}$.

We have

$P($ Layer $1, \ldots, i-1$ decoded, layer $i$ decoding failed $)$

$=P\left(\right.$ Layer $1, \ldots, i-1$ decoded, layer $i$ decoding failed $\mid X_{1}, \ldots, X_{i-1}$ available to decode layer $\left.i\right)$

$\leq P\left(\right.$ Layer $i$ decoding failed $\mid X_{1}, \ldots, X_{i-1}$ available to decode layer $\left.i\right)$

$\doteq \rho^{-d^{\star}\left(r_{i}, \gamma_{i-1}, \gamma_{i}\right)}$.

If $d^{\star}\left(r_{i}, \gamma_{i-1}, \gamma_{i}\right)>0$ for all $i$, then,

$P($ All layers decoded $)=1-\sum_{i} P\left(\right.$ Layer $1, \ldots, i-1$ decoded, layer $i$ decoding failed $\doteq \rho^{0}$.

From (18), (19), and (20) we have

$$
D \doteq \sum_{i=1}^{N_{s}} \rho^{-\left(b \sum_{j=1}^{i-1} r_{j}+d^{*}\left(r_{i}, \gamma_{i-1}, \gamma_{i}\right)\right)}+\rho^{-b \sum_{i=1}^{N_{s}} r_{i}}
$$

Let

$$
a(i)=b \sum_{j=1}^{i-1} r_{j}+d^{*}\left(r_{i}, \gamma_{i-1}, \gamma_{i}\right)
$$

be the exponent corresponding to the case when the $i$ th layer is in outage and $a\left(N_{s}+1\right)=b \sum_{i=1}^{N_{s}} r_{i}$ the exponent when all layers are decoded. From (21), the distortion SNR exponent for the broadcast scheme is

$$
a_{B S}(b)=\max _{r, \gamma} \min _{i} a(i)
$$

In the following proof, we fix $r_{i}=(k+1)\left(\gamma_{i-1}-\gamma_{i}\right)-\epsilon$ and optimize the power allocation $\gamma_{i}$ 's for $i=1$ to $N_{s}$ in order to maximize the exponent. Note that $\gamma_{0}=1$.

In section $\mathrm{A}$ of the Appendix, using the Karush-Kuhn-Tucker (KKT) conditions, it is proved that for $b>\frac{(M-k-1)(N-k-1)}{k+1}$ the optimal exponent is obtained when all the exponents $a(i)$ are equal provided that the resulting solution satisfies $\gamma_{i}>\gamma_{i+1}$ for all $i$ and $\gamma_{N_{s}}>0$.

In order for $a(i)=a(i+1)$, from (22) we have

$$
d^{*}\left(r_{i}, \gamma_{i-1}, \gamma_{i}\right)=b r_{i}+d^{*}\left(r_{i+1}, \gamma_{i}, \gamma_{i+1}\right)
$$


Since $r_{i}$ is chosen to be $(k+1)\left(\gamma_{i-1}-\gamma_{i}\right)-\epsilon$, from (7) we have

$$
d^{*}\left(r_{i}, \gamma_{i-1}, \gamma_{i}\right)=(M-k)(N-k) \gamma_{i-1}-(M+N-1-2 k)\left(\gamma_{i-1}-\gamma_{i}-\epsilon\right) .
$$

Substituting from (25) in (24) and using $r_{i}=(k+1)\left(\gamma_{i-1}-\gamma_{i}\right)$ we have

$$
\begin{aligned}
& (M-k)(N-k) \gamma_{i-1}-(M+N-1-2 k)\left(\gamma_{i-1}-\gamma_{i}\right) \\
& \quad=b(k+1)\left(\gamma_{i-1}-\gamma_{i}\right)+(M-k)(N-k) \gamma_{i}-(M+N-1-2 k)\left(\gamma_{i}-\gamma_{i+1}\right)+O(\epsilon) .
\end{aligned}
$$

On simplifying we obtain

$$
\left(\gamma_{i}-\gamma_{i+1}\right)=\alpha\left(\gamma_{i-1}-\gamma_{i}\right)+O(\epsilon)
$$

where

$$
\alpha=\frac{b(k+1)-(M-k-1)(N-k-1)}{M+N-1-2 k} .
$$

We can use (26) recursively to obtain

$$
\gamma_{i}-\gamma_{i+1}=\alpha^{i}\left(\gamma_{0}-\gamma_{1}\right)+O(\epsilon)=\alpha^{i}\left(1-\gamma_{1}\right)+O(\epsilon)
$$

Therefore,

$$
1-\gamma_{i}=\sum_{j=1}^{i}\left(\gamma_{j-1}-\gamma_{j}\right)=\sum_{j=1}^{i} \alpha^{j-1}\left(1-\gamma_{1}\right)+O(\epsilon)=\frac{1-\alpha^{i}}{1-\alpha}\left(1-\gamma_{1}\right)+O(\epsilon)
$$

Furthermore, if $b \sum_{j=1}^{N_{s}} r_{j}=a(1)$, we have

$$
\begin{aligned}
b \sum_{j=1}^{N_{s}}(k+1)\left(\gamma_{j-1}-\gamma_{j}\right) & =(M-k)(N-k)-(M+N-1-2 k)\left(1-\gamma_{1}\right)+O(\epsilon) \\
\Rightarrow b(k+1) \frac{1-\alpha^{N_{s}}}{1-\alpha}\left(1-\gamma_{1}\right) & =(M-k)(N-k)-(M+N-1-2 k)\left(1-\gamma_{1}\right)+O(\epsilon) \\
\Rightarrow\left(1-\gamma_{1}\right) & =\frac{(M-k)(N-k)(1-\alpha)}{b(k+1)\left(1-\alpha^{N_{s}}\right)+(M+N-1-2 k)(1-\alpha)} .
\end{aligned}
$$

From (27) we have

$$
\left(1-\gamma_{1}\right)=\frac{(M-k)(N-k)(1-\alpha)}{(M-k)(N-k)-b(k+1) \alpha^{N_{s}}}
$$

From (29)

$$
\begin{aligned}
\left(1-\gamma_{i}\right) & =\frac{(M-k)(N-k)\left(1-\alpha^{i}\right)}{(M-k)(N-k)-b(k+1) \alpha^{N_{s}}} \\
\gamma_{i} & =\frac{(M-k)(N-k) \alpha^{i}-b(k+1) \alpha^{N_{s}}}{(M-k)(N-k)-b(k+1) \alpha^{N_{s}}} .
\end{aligned}
$$

Consider the case when $0 \leq \alpha \leq 1$, i.e., when $\frac{(M-k-1)(N-k-1)}{k+1} \leq b \leq \frac{(M-k)(N-k)}{k+1}$. Since $1 \geq \alpha^{i}$ and since $(M-k)(N-k) /(b(k+1))>1>\alpha^{N_{s}}$, from (31) it follows that $\gamma_{i} \leq 1$. From (32), since $(M-k)(N-k) /(b(k+1))>1>\alpha^{N_{s}-i}$, we have $\gamma_{i} \geq 0$ and we also observe that $\gamma_{i}$ is a decreasing sequence in $i$. Therefore, this a valid power allocation.

The resulting exponent is $b(k+1) \frac{(M-k)(N-k)\left(1-\alpha^{N_{s}}\right)}{(M-k)(N-k)-b(k+1) \alpha^{N_{s}}}$ and on taking the limit as $N_{s} \rightarrow \infty$ we obtain $b(k+1)$. 


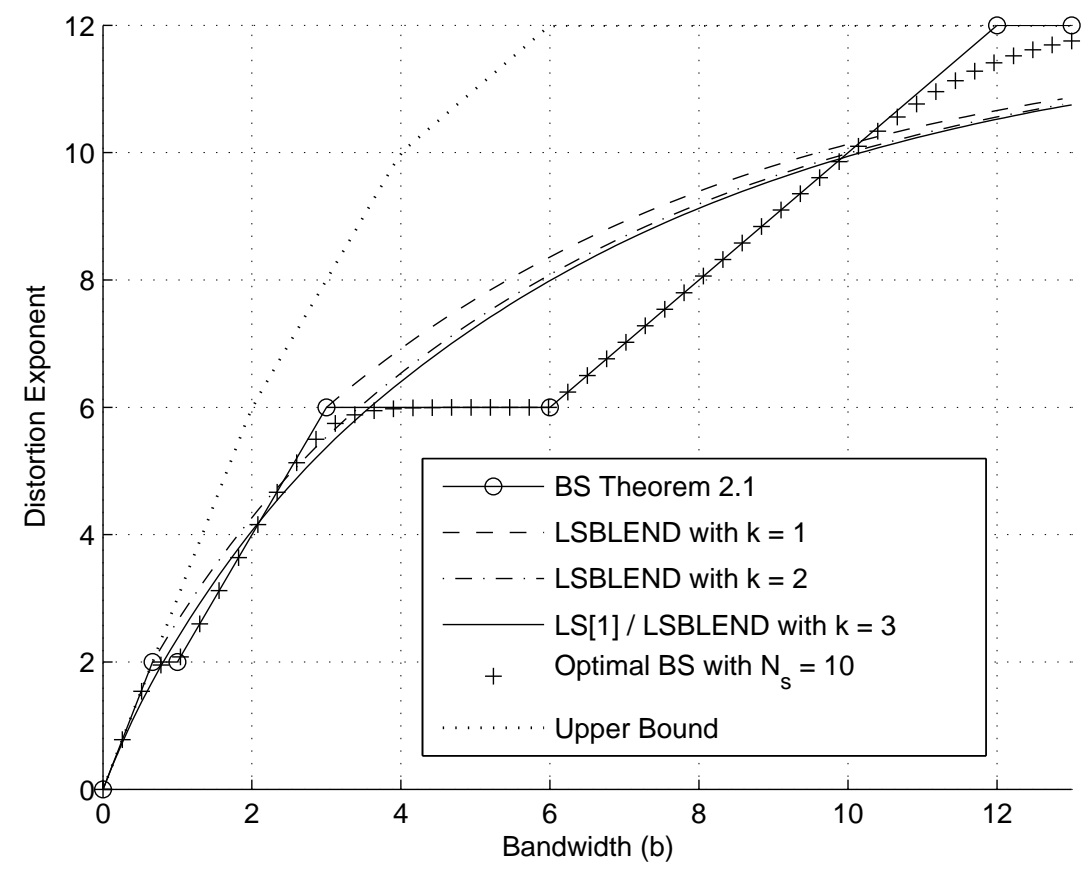

Fig. 3. Distortion SNR exponent for $M=3, N=4$

For the region $\frac{(M-k)(N-k)}{k+1} \leq b \leq \frac{(M-k)(N-k)}{k}$, Theorem 2.1 does not specify any achievable exponent. But notice that the exponent corresponding to both $b=\frac{(M-k)(N-k)}{k+1}$ and $b=\frac{(M-k)(N-k)}{k}$ is $(M-k)(N-k)$. For this region, we can ignore the additional bandwidth $b-\frac{(M-k)(N-k)}{k+1}$ and use a power allocation corresponding to $b=\frac{(M-k)(N-k)}{k}$ to achieve an exponent of $(M-k)(N-k)$. The resulting achievable distortion SNR exponent curve is continuous and is flat in the region $\frac{(M-k)(N-k)}{k+1} \leq b \leq \frac{(M-k)(N-k)}{k}$ for $k=1$ to $m-1$. and for $b>M N$.

Corollary 2.1: The optimal distortion SNR exponent for $b<(n-m+1) / m$ is $m b$.

Proof: The result is obtained by comparing the upper bound in (5) with the achievable exponent specified in Theorem 2.1 for the case when $k=(m-1)$.

For $b<(n-m+1) / m$ and $b>m n$, BS achieves the optimal exponent (it matches the informed transmitter upper bound) and hence the power and rate allocation specified in Theorem 2.1 is optimal. For the region between these two values, the next two results prove that the exponent achieved in Theorem 2.1 is the optimal exponent achievable by any superposition (broadcast) scheme. This is shown by finding an upper bound to the exponent of any superposition scheme, for any power allocation and number of layers, that matches the achievable exponent of Theorem 2.1. This also calls for schemes that are not based on superposition alone in order to improve on the achievable exponent in this region (discussed in the next sections).

Theorem 2.2: For $b \leq \frac{(M-k)(N-k)}{k}$, the distortion SNR exponent of the broadcast scheme $a_{B S}(b) \leq$ $(M-k)(N-k)$.

Proof: Recall that the exponent of the broadcast scheme is given by $a_{B S}(b)=\min _{i} a(i)$ where $a(i)$ 


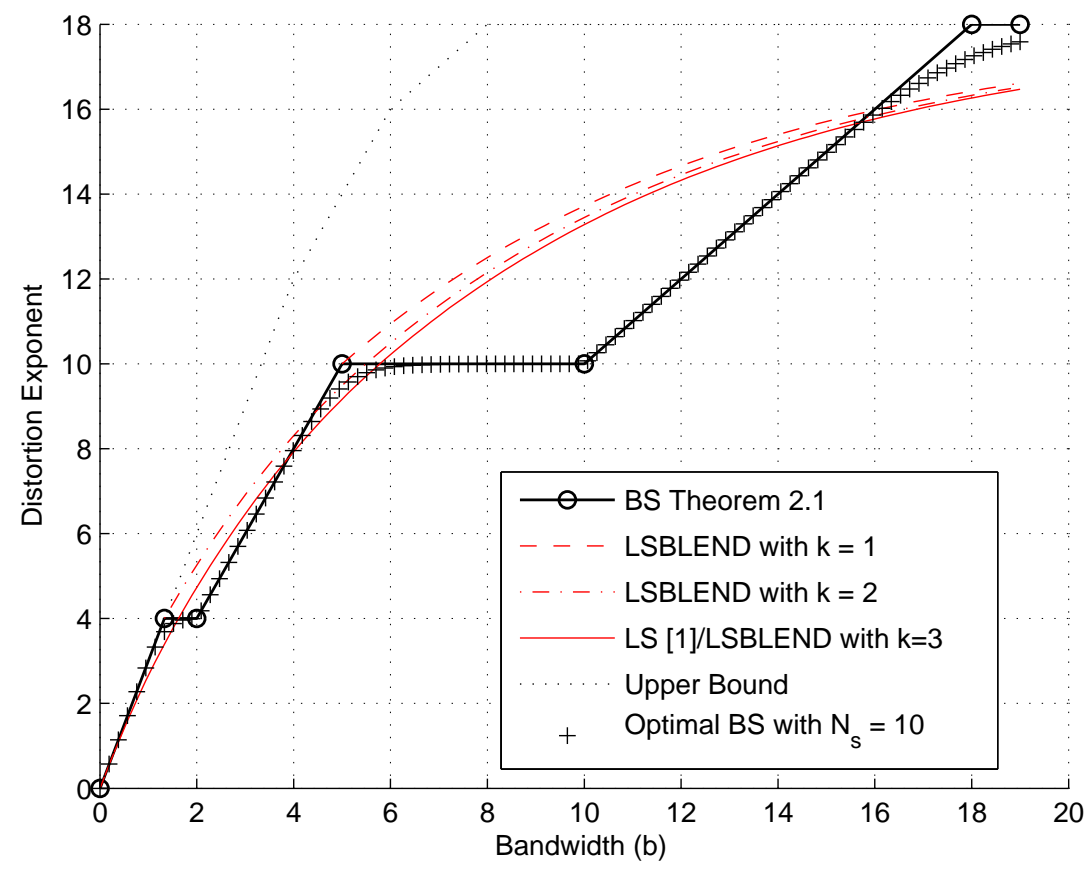

Fig. 4. Distortion SNR exponent for $M=3, N=6$

is as specified in (22).

Let us fix $b$ and $k$ such that $(M-k)(N-k) / k \geq b$. Let us assume that there exists a power and rate allocation such that the exponent $a_{B S}(b)>(M-k)(N-k)$, then since $a_{B S}(b)=\min _{i} a(i)$, then for all $i$ from 1 to $N_{s}+1$ we must have $a(i)>(M-k)(N-k)$. As before, without loss of generality, let the rate used in the $i$ th layer be $r_{i}=k_{i}\left(\gamma_{i-1}-\gamma_{i}\right)+\delta_{i}$, for some integer $k_{i}$ and $0 \leq \delta_{i}<\gamma_{i-1}-\gamma_{i}$. We will now show that if $a_{B S}(b)>(M-k)(N-k)$ were to be true, then $k_{i}<k$ for all $i$.

The gist of the proof is as follows. If, to the contrary, $k_{i} \geq k$ for some $i$, then there must be a smallest value of $i$ (say $i^{*}$ ) for which this is true. That is, there must be an $i^{*} \geq 1$, for which $k_{i^{*}} \geq k$ and $k_{i} \leq k-1$ for all $i=1$ to $i^{*}-1$. We will now show that $a\left(i^{*}\right)$ cannot be larger than $(M-k)(N-k)$.

We have

$$
a\left(i^{*}\right)=b \sum_{i=1}^{i^{*}-1} r_{i}+d^{*}\left(r_{i^{*}}, \gamma_{i^{*}-1}, \gamma_{i^{*}}\right)
$$


Since, $r_{i}=k_{i}\left(\gamma_{i-1}-\gamma_{i}\right)+\delta_{i}$, clearly $r_{i} \leq\left(k_{i}+1\right)\left(\gamma_{i-1}-\gamma_{i}\right)$. Therefore,

$$
\begin{aligned}
a\left(i^{*}\right) & \leq b \sum_{i=1}^{i^{*}-1}\left(k_{i}+1\right)\left(\gamma_{i-1}-\gamma_{i}\right)+d^{*}\left(r_{i^{*}}, \gamma_{i^{*}-1}, \gamma_{i^{*}}\right) \\
& \leq b \sum_{i=1}^{i^{*}-1}(k)\left(\gamma_{i-1}-\gamma_{i}\right)+d^{*}\left(r_{i^{*}}, \gamma_{i^{*}-1}, \gamma_{i^{*}}\right) \quad\left(\because k_{i} \leq k-1, \text { for } i<i^{*}\right) \\
& =b k\left(1-\gamma_{i^{*}-1}\right)+d^{*}\left(r_{i^{*}}, \gamma_{i^{*}-1}, \gamma_{i^{*}}\right) \\
& \leq(M-k)(N-k)\left(1-\gamma_{i^{*}-1}\right)+d^{*}\left(r_{i^{*}}, \gamma_{i^{*}-1}, \gamma_{i^{*}}\right) \quad(\because b \leq(M-k)(N-k) / k) \\
& \leq(M-k)(N-k)\left(1-\gamma_{i^{*}-1}\right)+\left(M-k_{i^{*}}\right)\left(N-k_{i^{*}}\right) \gamma_{i^{*}-1} \quad\left(\because \delta_{i^{*}} \geq 0\right) \\
& \leq(M-k)(N-k)\left(1-\gamma_{i^{*}-1}\right)+(M-k)(N-k) \gamma_{i^{*}-1} \quad\left(\because k_{i^{*}} \geq k\right) \\
& =(M-k)(N-k) .
\end{aligned}
$$

For $a_{B S}>(M-k)(N-k)$, we require $a(i)>(M-k)(N-k), \forall i$ and, hence, we must have that $k_{i} \leq k-1$, for all $i=1, \ldots, N_{s}$. This implies that $r_{i} \leq k\left(\gamma_{i-1}-\gamma_{i}\right)$. But, in this case,

$$
a\left(N_{s}+1\right)=b \sum_{i=1}^{N_{s}} r_{i} \leq b k\left(1-\gamma_{N_{s}}\right) \leq(M-k)(N-k) .
$$

Therefore, our assumption that $a_{B S}(b)$ can be greater than $(M-k)(N-k)$ for $b<(M-k)(N-k) / k$ is not valid. Hence proved.

As pointed out in the discussion after Theorem 2.1, the achievable exponent for $(M-k)(N-k) /(k+1) \leq$ $b \leq(M-k)(N-k) / k$ is $(M-k)(N-k)$. This combined with the upper bound specified in Theorem 2.2 proves that this is the best achievable exponent using any broadcast scheme for this range of $b$.

Theorem 2.3: For $b>(M-k-1)(N-k-1) /(k+1)$ the distortion SNR exponent of the broadcast scheme $a_{B S}(b) \leq b(k+1)$.

Proof: Recall that the exponent of the broadcast scheme is given by $a_{B S}(b)=\min _{i} a(i)$ where $a(i)$ is as specified in (22). The idea of the proof is similar to that in the proof of the previous theorem. Again we fix $b$ and $k$ such that $b>(M-k-1)(N-k-1) /(k+1)$. Let us assume that there exists a power and rate allocation policy such that $a_{B S}(b)>b(k+1)$. Let the rate allocation be $r_{i}=k_{i}\left(\gamma_{i-1}-\gamma_{i}\right)+\delta_{i}$ as before.

The proof is similar to that of the previous theorem and is to first show that $k_{i} \leq k$ for all $i$. As before, let $i^{*} \geq 1$ be such that Let $k_{i} \leq k$ for $i=1$ to $i^{*}-1$ and $k_{i^{*}} \geq k+1$. We have

$$
\begin{aligned}
a\left(i^{*}\right)= & b \sum_{i=1}^{i^{*}-1} r_{i}+d^{*}\left(r_{i^{*}}, \gamma_{i^{*}-1}, \gamma_{i^{*}}\right) \\
\leq & b(k+1)\left(1-\gamma_{i^{*}-1}\right)+\left(M-k_{i^{*}}\right)\left(N-k_{i^{*}}\right) \gamma_{i^{*}-1} \\
& \quad\left(\because r_{i} \leq\left(k_{i}+1\right)\left(\gamma_{i-1}-\gamma_{i}\right) \leq(k+1)\left(\gamma_{i-1}-\gamma_{i}\right) \text { for } i<i^{*}\right) \\
\leq & b(k+1)\left(1-\gamma_{i^{*}-1}\right)+(M-k-1)(N-k-1) \gamma_{i^{*}-1} \quad\left(\because k_{i^{*}} \geq k+1\right) \\
= & b(k+1)-\gamma_{i^{*}-1}(b(k+1)-(M-k-1)(N-k-1)) \\
\leq & b(k+1) \quad(\because b>(M-k-1)(N-k-1) /(k+1)) .
\end{aligned}
$$


This contradicts the assumption that $a_{B S}(b)>b(k+1)$. Therefore, the only other possibility is that $k_{i} \leq k$ for all $i$. In this case too, $a\left(N_{s}+1\right)=b \sum_{i=1}^{N_{s}} r_{i} \leq b(k+1)$ which implies that the assumption $a_{B S}(b)>b(k+1)$ is incorrect. Hence proved.

Note that for $(M-k-1)(N-k-1) /(k+1) \leq b \leq(M-k)(N-k) /(k+1)$ the achievable exponent in Theorem 2.1 is also $b(k+1)$. Hence, Theorem 2.1 along with Theorems 2.2 and 2.3 fully characterize the exponent achievable with any broadcast scheme.

Finite Number of Layers: In practice it is not possible to have infinitely many layers and it is important to study the performance of the broadcast scheme with a finite number of layers. The problem of finding the optimal distortion SNR exponent for a finite number of layers can be posed as the following optimization problem.

$$
\begin{array}{cl}
\max & a \\
\text { subject: } & \text { for all } i \in\left\{1,2, \ldots, N_{s}\right\} \\
& \gamma_{i} \geq 0, \delta_{i} \geq 0, r_{i} \geq 0, k_{i} \in\{0,1, \ldots, m-1\} \\
& \gamma_{i-1}>\gamma_{i}, \gamma_{0}=1 \\
& \delta_{i}<\gamma_{i-1}-\gamma_{i} \\
& r_{i}=k_{i}\left(\gamma_{i-1}-\gamma_{i}\right)+\delta_{i} \\
& a \leq b \sum_{j=1}^{i-1} r_{j}+\left(m-k_{i}\right)\left(n-k_{i}\right) \gamma_{i-1}-\left(m+n-1-2 k_{i}\right) \delta_{i}, \\
& a \leq b \sum_{j=1}^{N_{s}} r_{j} .
\end{array}
$$

For a fixed set of $k_{i}$ 's this reduces to a linear program. For small $N_{s}$, the optimum exponent can be found by using the linear program for all $m^{N_{s}}$ choices of $k_{i}$ 's .

In Fig. 3 and Fig. 4, the distortion SNR exponent corresponding to the broadcast scheme proposed in Theorem 2.1 is shown for a $3 \times 4$ and a $3 \times 6$ MIMO system. The optimal distortion SNR exponent corresponding to the broadcast scheme with 10 layers is also shown. We see that the exponent with finite layers is very close to the best achievable distortion exponent of the broadcast scheme for all $b$ and the curves overlap for a large range of $b$. Also as proved in Theorem 2.2 and 2.3 , the distortion exponent with finite layers does not improve on the achievable exponent specified in Theorem 2.1 .

\section{B. Layering in Time with one Broadcast layer at the end}

Consider the scheme shown in Fig. 5. For $b>b_{k}=(m-k)(n-k) /(k+1), k \in\{1, \ldots, m\}$, a bandwidth of $b-b_{k}$ is allocated for time layering and the remaining bandwidth of $b_{k}$ is allocated to a broadcast scheme where the rate and power allocation for the broadcast layers are chosen as specified in Theorem 2.1. The parameter $k$ determines the bandwidth splitting between the broadcast layer and the time layers. The decoding proceeds by first decoding the time layers and then decoding the broadcast layers after all the time layers are decoded. This is similar to the HDA scheme of [5] and the HLS of [1] where the 


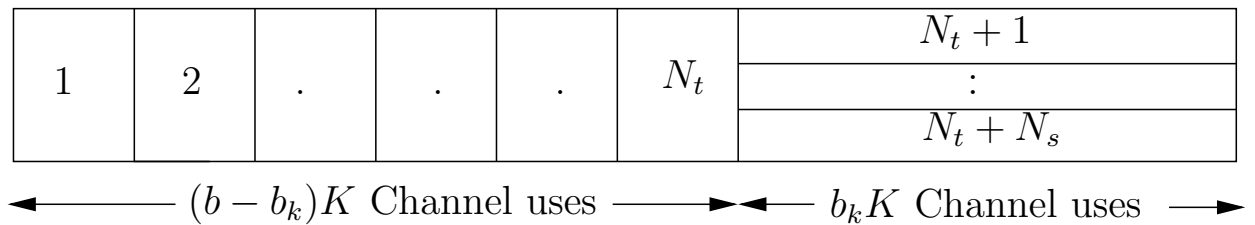

Fig. 5. Layered Schemes with Broadcast Layer at the end (LSBLEND)

source is quantized and transmitted using time layering in a bandwidth of $b-1 / m$ and the quantization error is transmitted in an analog layer of bandwidth $1 / \mathrm{m}$. For the proposed scheme, the distortion SNR exponent obtained for a particular bandwidth splitting parameter $k$ is given in the following theorem. The largest achievable distortion SNR exponent is then obtained by taking a supremum over all $k$.

Note that when $k=m$, the bandwidth allocated to the broadcast layer is 0 , i.e., we have only time layering. This scheme, termed Layered Scheme (LS), was proposed and analyzed in [1]. The proof of the following theorem is similar to the derivation of the exponent for LS in [1].

Theorem 2.4: Let $c_{j}=(m+n-1-2 j) \log \frac{j+1}{j}$ for $j=0, \ldots, m$. Let $p$ be such that $\sum_{j=p+1}^{k-1} c_{j} \leq$ $\left(b-b_{k}\right)<\sum_{j=p}^{k-1} c_{j}$. Then, the best distortion SNR exponent $a(b)$ achievable using LSBLEND is given by

$$
a(b)=m n-p-p^{2}-(m+n-1-2 p)(p+1) e^{-\frac{b-b_{k}-\sum_{j-p+1}^{k-1} c_{j}}{m+n-1-2 p}} .
$$

Proof: Let $N_{t}$ and $N_{s}$ denote the number of time and superposition layers respectively. Let $a(i)$ for $i=1, \ldots, N_{t}$ denote the distortion SNR exponent corresponding to the case when the time layers 1 to $i-1$ are decoded and decoding of the $i$ th time layer fails, $a\left(N_{t}+i\right)$ for $i=1, \ldots, N_{s}$ denote the distortion SNR exponent corresponding to the case when all $N_{t}$ time layers and the first $i-1$ broadcast layers are decoded while decoding of the $i$ th broadcast layer fails, and let $a\left(N_{t}+N_{s}+1\right)$ denote the exponent corresponding to the case when all layers are decoded.

For the $i$ th time layer, the probability of decoding failure is given by $P_{e}(i) \doteq \rho^{-d^{*}\left(r_{i}\right)}$ where $r_{i}$ is the multiplexing rate of the $i$ th time layer and $d^{*}\left(r_{i}\right)$ is the Zheng and Tse diversity multiplexing tradeoff function specified in (4). Note that power allocation to the time layer is $\rho^{1}-\rho^{0}$ and $d^{*}\left(r_{i}\right)=d^{*}\left(r_{i}, 1,0\right)$. The bandwidth allocated to a time layer is $\left(b-b_{k}\right) / N_{t}$. The distortion SNR exponent of the $i$ th time layer is then given by

$$
a(i)=\frac{b-b_{k}}{N_{t}} \sum_{j=0}^{i-1} r_{j}+d^{*}\left(r_{i}\right)
$$

where $r_{0}=0$.

For the broadcast layer we use the rate and power allocation as specified in Theorem 2.1. With that rate and power allocation it follows that the exponents $a\left(N_{t}+1\right), a\left(N_{t}+2\right), \ldots a\left(N_{t}+N_{s}+1\right)$ are all 
equal and are given by

$$
\begin{aligned}
a\left(N_{t}+i\right) & =\frac{b-b_{k}}{N_{t}} \sum_{j=0}^{N_{t}} r_{j}+(k+1) b_{k} \\
& =\frac{b-b_{k}}{N_{t}} \sum_{j=0}^{N_{t}} r_{j}+(m-k)(n-k)
\end{aligned}
$$

for $i=1$ to $N_{s}+1$ in the limit $N_{s} \rightarrow \infty$. Note that we do not loose optimality here by fixing the rate and power allocation of the broadcast layer since $(m-k)(n-k)$ is the maximum possible contribution that the broadcast layer of bandwidth $b_{k}$ can make to the exponent (see Theorem 2.2 and Theorem 2.3).

In the following proof, we optimize $r_{i}$ 's to maximize the distortion SNR exponent.

In section $\mathrm{B}$ of the Appendix we show that the exponent is maximized by choosing $r_{i}$ 's such that $a(1)=a(2)=\ldots=a\left(N_{t}\right)=a\left(N_{t}+1\right)$ provided that the resulting $r_{i}$ 's lie between 0 and $m$. By setting $a\left(N_{t}\right)=a\left(N_{t}+1\right)$ we obtain

$$
d^{*}\left(r_{N_{t}}\right)=\frac{b-b_{k}}{N_{t}} r_{N_{t}}+(m-k)(n-k)
$$

We will consider the limiting case when $N_{t} \rightarrow \infty$. From (38) we have, in the limiting case,

$$
d^{*}\left(r_{N_{t}}\right) \rightarrow(m-k)(n-k) .
$$

Therefore,

$$
\left(m-k_{N_{t}}\right)\left(n-k_{N_{t}}\right)-\left(m+n-1-2 k_{N_{t}}\right) \delta_{N_{t}} \rightarrow(m-k)(n-k) .
$$

This happens when $k_{N_{t}}=k-1$ and $\delta_{N_{t}} \rightarrow 1$.

By setting $a(i-1)=a(i)$ we have

$$
d^{*}\left(r_{i-1}\right)=\frac{b-b_{k}}{N_{t}} r_{i-1}+d^{*}\left(r_{i}\right)
$$

$d^{*}(r)$ is a decreasing function and from (41) we have $d^{*}\left(r_{i-1}\right) \geq d^{*}\left(r_{i}\right)$. Therefore $r_{N_{t}} \geq r_{N_{t}-1} \geq \ldots \geq r_{1}$. Let $r_{i}$ lie between $t$ and $t+1$. We want to check if $r_{i-1}$ also lies between $t$ and $t+1$. To do so we assume that $k_{i-1}=t$ and solve for $\delta_{i-1}$. If the resulting $\delta_{i-1}$ lies between 0 and 1 , then the assumption $k_{i}=t$ is correct. From (41) we have

$$
\begin{aligned}
(m-t)(n-t)-(m+n-1-2 t) \delta_{i-1} & =\frac{b-b_{k}}{N_{t}}\left(t+\delta_{i-1}\right)+(m-t)(n-t)-(m+n-1-2 t) \delta_{i} . \\
\Rightarrow \delta_{i-1}\left(m+n-1-2 t+\frac{b-b_{k}}{N_{t}}\right) & =\delta_{i}(m+n-1-2 t)-\frac{b-b_{k}}{N_{t}} t . \\
\Rightarrow \delta_{i-1} & =\alpha \delta_{i}-(1-\alpha) t
\end{aligned}
$$

where

$$
\alpha=\frac{m+n-1-2 t}{m+n-1-2 t+\left(b-b_{k}\right) / N_{t}}<1 .
$$

On using recursion (42) $N_{l}$ times we have

$$
\delta_{i-N_{l}}=\alpha^{N_{l}} \delta_{i}-\frac{1-\alpha^{N_{l}}}{1-\alpha}(1-\alpha) t=\alpha^{N_{l}}\left(t+\delta_{i}\right)-t .
$$


The maximum number of times the recursion can be used such that the resulting $\delta$ is positive is given by

$$
\begin{aligned}
\alpha^{N_{l}}\left(t+\delta_{i}\right) & \geq t \\
\Rightarrow N_{l} \log \alpha & \geq \log \frac{t}{t+\delta_{i}} \\
\Rightarrow \frac{N_{l}}{N_{t}} & \leq \frac{1}{N_{t} \log \alpha} \log \frac{t}{t+\delta_{i}} \quad \because \alpha<1, \log \alpha<0 \\
\Rightarrow \frac{N_{l}}{N_{t}} & \leq-\frac{1}{\log \left(\left(1+\frac{b-b_{k}}{(m+n-1-2 t) N_{t}}\right)^{N_{t}}\right)} \log \frac{t}{t+\delta_{i}} \\
\Rightarrow \frac{N}{N_{t}} & \leq \frac{m+n-1-2 t}{b-b_{k}} \log \frac{t+\delta_{i}}{t} \quad\left(N_{t} \rightarrow \infty\right) .
\end{aligned}
$$

For the proposed scheme, we start from $r_{N_{t}}=k\left(k_{N_{t}}=k-1\right.$ and $\left.\delta_{N_{t}}=1\right)$ and solve for $r_{i-1}$ from $r_{i}$. Let $c_{j}=(m+n-1-2 j) \log \frac{j+1}{j}$. If $p$ is such that $\sum_{j=p+1}^{k-1} c_{j} \leq\left(b-b_{k}\right)<\sum_{j=p}^{k-1} c_{j}$ then as $i$ decreases from $N_{t}$, after a fraction $\sum_{j=p+1}^{k-1} c_{j} /\left(b-b_{k}\right)$ of the time layers, $r_{i}$ decreases from $k$ to $p+1$. For the remaining fraction $\left(1-\sum_{j=p+1}^{k-1} c_{j} /\left(b-b_{k}\right)\right)$ of layers, as $i$ decreases, $r_{i}$ decreases but remains above $p$, i.e., $k_{i}$ remains constant at $p$ while $\delta_{i}$ decreases. From (44) we can calculate $r_{1}$ as

$$
\begin{aligned}
r_{1} & =p+\lim _{N_{t} \rightarrow \infty} \alpha^{N_{t}\left(1-\frac{1}{b-b_{k}} \sum_{j=p+1}^{k-1} c_{j}\right)}(p+1)-p \\
& =(p+1) e^{-\left(\frac{b-b_{k}}{m+n-1-2 p}\right)\left(1-\frac{1}{b-b_{k}} \sum_{j=p+1}^{k-1} c_{j}\right)} .
\end{aligned}
$$

The final exponent is given by

$$
\begin{aligned}
a(1)=d^{*}\left(r_{1}\right) & =(m-p)(n-p)-(m+n-1-2 p)\left(r_{1}-p\right) \\
& =m n-p-p^{2}-(m+n-1-2 p) r_{1}
\end{aligned}
$$

which is the desired result.

Note that when $m=n$ and $k=m-1$, the contribution to the distortion SNR exponent by the broadcast layer is $b_{k}(k+1)=1$ and it uses a bandwidth of $b_{k}=1 / m$. In the HLS scheme, the analog layer uses a bandwidth of $b_{0}=1 / m$ and it also has a contribution of $m b_{0}=1$ towards the exponent. Therefore, in this case, the distortion SNR exponent obtained with LSBLEND with $k=m-1$ is identical to that with HLS. Therefore, the distortion SNR exponent obtained using LSBLEND becomes identical to that obtained using HLS when (a) $m=n$ and (b) the supremum occurs at $k=m-1$. It can be shown that LSBLEND is strictly better otherwise for $b>1 / m$.

\section{Digital Layering in Time and Using Superposition}

The source is encoded in such a way that it is successively refinable. The transmitted signal composes of $N_{t}$ time layers where each time layer is a superposition of $N_{s}$ layers. To the $(i, j)$ th layer, i.e., the $j$ th time layer and the $i$ th superposition layer within it, we allocate a power level of $\rho^{\gamma_{i-1, j}}-\rho^{\gamma_{i, j}}$ and we use a rate of transmission of $r_{i, j} \log \rho$. This corresponds to a source coding rate of $\left(b / N_{t}\right) r_{i, j} \log \rho$. The order in which the source coded bits are mapped to the transmission layers is important. The source coded 


\begin{tabular}{|c|c|c|c|c|c|c|c|c|c|}
\hline 1 & 2 & 3 & & & & & & & $\mathrm{~N}_{\mathrm{t}}$ \\
\hline $\mathrm{N}_{\mathrm{t}}+1$ & $\mathrm{~N}_{\mathrm{t}}+2$ & & & & & & & & \\
\hline & & & & & & & & & $\mathrm{N}_{\mathrm{t}} \mathrm{N}_{\mathrm{S}}$ \\
\hline
\end{tabular}

Fig. 6. Box Scheme

bits are successively mapped on to the transmitted layers from top left to bottom right going along each row. That is, in the order $(1,1), \ldots,\left(1, N_{t}\right),(2,1), \ldots,\left(2, N_{t}\right), \ldots,\left(N_{s}, N_{t}\right)$ (see Fig. 6). The decoding proceeds in the same order and when a layer cannot be decoded, the source is reconstructed using all the layers that have been successfully decoded up to that layer.

Let $\bar{r}_{(i-1) N_{t}+j} \log \rho=\left(b / N_{t}\right)\left(\sum_{p=1}^{i-1} \sum_{q=1}^{N_{t}} r_{p, q}+\sum_{q=1}^{j-1} r_{i, q}\right) \log \rho$ denote the cumulative source coding rate up to the $(i, j)$ th layer. As in the broadcast scheme case, we can approximate the overall distortion up to an exponential order by

$$
D \doteq \sum_{i} \sum_{j} \rho^{-d^{\star}\left(r_{i, j}, \gamma_{i-1, j}, \gamma_{i, j}\right)+\bar{r}_{(i-1) N_{t}+j}}+\rho^{-\bar{r}_{N_{s} N_{t}+1}}
$$

Let $r$ and $\gamma$ denote the set of $r_{i, j}$ 's and $\gamma_{i, j}$ 's. For a given $r, \gamma$, the overall exponent of the scheme $a(b, r, \gamma)$ is then,

$$
a(b, r, \gamma)=\min _{i, j}\left(d^{\star}\left(r_{i, j}, \gamma_{i-1, j}, \gamma_{i, j}\right)+\bar{r}_{(i-1) N_{t}+j}, \bar{r}_{N_{s} N_{t}+1}\right)
$$

The best achievable exponent with this scheme $a(b)$ is then given by

$$
a(b)=\max _{r, \gamma} a(b, r, \gamma) .
$$

If we allow for change in the bandwidth allocated to each layer, then both BS and LSBLEND become special cases of this scheme and therefore the exponent obtained from the maximization should be better than those reported earlier. We will now show that for the distortion SNR exponent, even with fixed bandwidth allocation to each layer, the Box scheme can be designed to perform at least as well as BS and LSBLEND.

Claim 2.1: The Box scheme with $N_{s}$ superposition layer and $N_{t}$ time layers has a distortion SNR exponent that is at least as good as that of the broadcast scheme with $N_{s}$ layers.

Proof: Let the optimal power allocation for the broadcast scheme by $r_{i}, \gamma_{i}$. The exponent corresponding to the $i$ th broadcast layer is $a_{B S}(i)=b \sum_{j=1}^{i-1} r_{j}+d^{*}\left(r_{i}, \gamma_{i-1}, \gamma_{i}\right)$. Now consider the Box scheme where the power allocation to the $(i, j)$ th layer $\gamma_{i, j}$ is set to $\gamma_{i}$ and the rate $r_{i, j}=r_{i}$. Then $a_{\text {Box }}(i, j)=b \sum_{j=1}^{i-1} r_{j}+\frac{b}{N_{t}}(j-1) r_{i}+d^{*}\left(r_{i}, \gamma_{i-1}, \gamma_{i}\right)$. Clearly $\frac{b}{N_{t}} \sum_{i, j} r_{i, j}=b \sum r_{i}$ and $a_{B o x}(i, j) \geq a(i)$. Therefore, $\min _{i, j}\left(a_{B o x}(i, j), \frac{b}{N_{t}} \sum_{i, j} r_{i, j}\right) \geq \min _{i}\left(a_{B S}(i), b \sum r_{i}\right)$. In this case it is actually equal but if we optimize the power allocation of the box scheme it could possibly improve on the exponent.

Claim 2.2: In the limit as $N_{t} \rightarrow \infty$, the Box scheme has a distortion SNR exponent that is at least as good as that of LSBLEND.

Proof: Consider the case when $\left(b-b_{k}\right) / N_{t}=b / N_{t, \text { Box }}$ where $N_{t}, N_{t, \text { Box }}$ are positive integers. Consider a power and rate allocation for the box scheme that is identical to the LSBLEND scheme for 
first $N_{t}$ time layers. That is, the first $N_{t}$ time layers have no superposition layers and the rate is identical to that of LSBLEND. For the remaining $N_{t, B o x}-N_{t}$ layers we allocate power and rate with the procedure used in lemma 2.1 and therefore its contribution to the exponent is identical to the contribution of the broadcast layer of LSBLEND. Therefore, this has an exponent that is identical to that of LSBLEND. Again, by optimizing the power and rate allocation of the box scheme we could possibly improve the exponent.

For the case when $\frac{b}{b-b_{k}}$ is irrational, the result still holds because the achievable exponent with LSBLEND and Box scheme is a continuous function of $b$.

The maximization in (48) is difficult to perform analytically and very quickly becomes difficult to perform even numerically. The procedure described in Algorithm 1 has been used to find a suboptimal set of $r, \gamma$. Remarkably, it turns out that for a range of $b$, this achieves performance very close to the informed transmitter upper bound $a_{I T}(b)$. Furthermore, for the considered examples, this scheme performs nearly as well as currently known schemes for all $b$ while it is strictly better for some range of $b$.

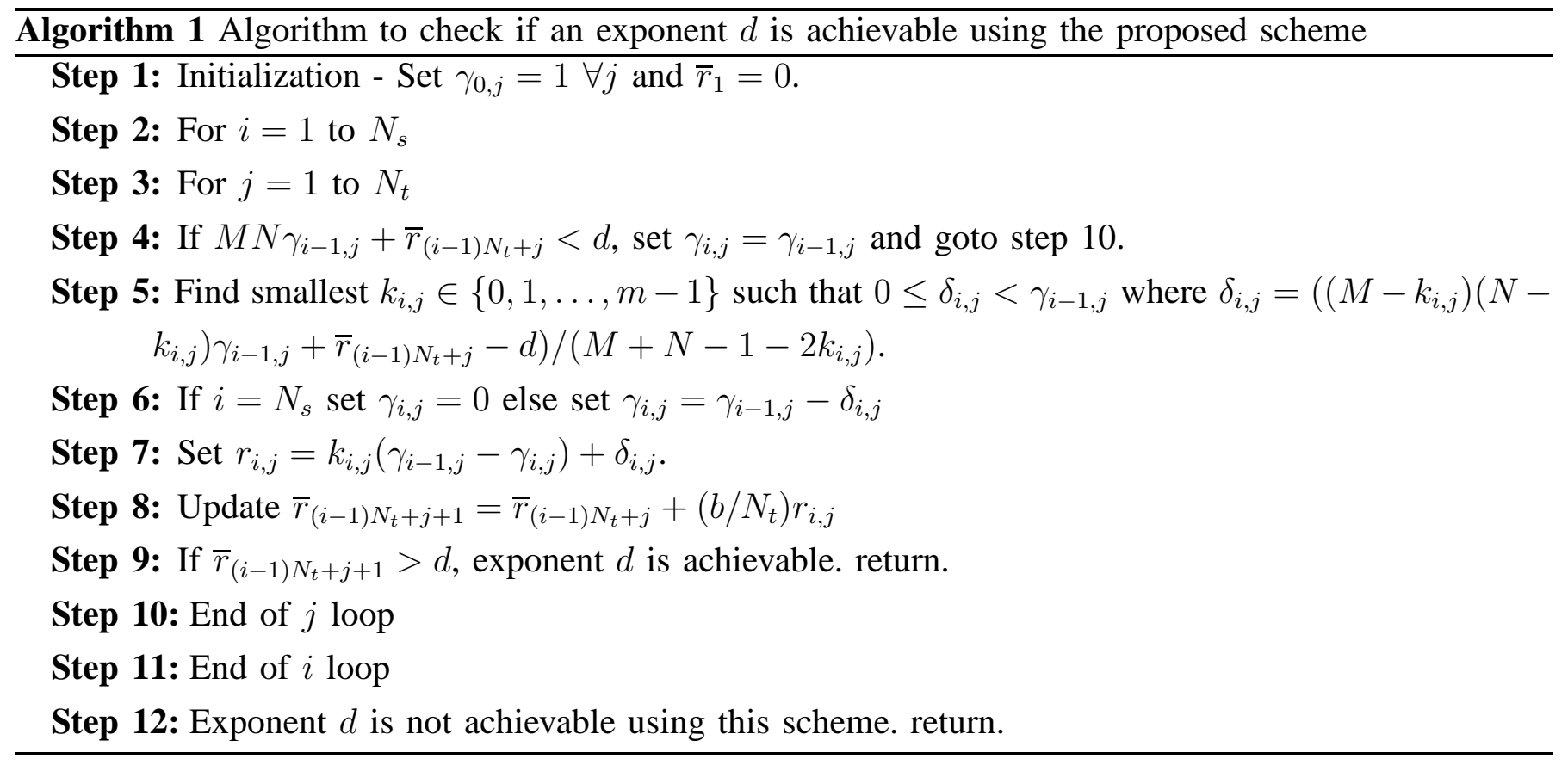

For each $(i, j)$ if we fix $k_{i, j} \in\{0,1, \ldots, m-1\}$ and let $r_{i, j}=k_{i, j}\left(\gamma_{i-1, j}-\gamma_{i, j}\right)+\delta_{i, j}$ where $0 \leq \delta_{i, j}<$ $\left(\gamma_{i-1, j}-\gamma_{i, j}\right)$, then, as before, the problem of finding the optimal exponent reduces to a linear program and hence by solving it for different $k_{i, j}$ 's we would expect to find an exponent that is better than that obtained using Algorithm 1. However, in Step 4 of the algorithm, notice that we skip a layer if it is not possible to allocate a non zero rate. Therefore, this layer is never in outage. However, in the linear program, if we use lemma 2.1 to compute $d^{*}\left(0, \gamma_{i-1, j}, \gamma_{i-1, j}\right)$ we get 0 which means this layer is always in outage. Therefore, to obtain the optimal exponent, we will need to allow for a layer to be skipped in addition to allowing for different values of $k$ for that layer. The complexity thus grows as $(M+1)^{N_{s} N_{t}}$.

The achievable exponent with this scheme increases monotonically with $N_{S}$. Interestingly, the achievable exponent with this scheme may not increase monotonically with $N_{t}$. 
We also considered the following variations, which provide some gain for finite number of layers. However, the gain diminishes as the number of layers increases.

1) Adding an Analog Layer: In this scheme, we start allocating rate and power levels to the layers as in Algorithm 1. Let us denote by $\mathcal{A}_{i, j}=\left\{(p, q): p \leq i-1\right.$ AND $\left.q \leq N_{t}\right\} \cup\{(i, q): q<j\}$ the set of all layers for which a rate and power allocation has been found during the $(i, j)$ th stage of the algorithm. Let $\mathbf{X}_{i, j}^{a}$ denote the analog quantization error in quantizing the source using $\bar{r}_{(i-1) N_{t}+j} \log \rho$ bits. We check if at least $\left\lceil\frac{N_{t}}{b m}\right\rceil$ layers are still available in $\mathcal{A}_{i, j}^{c}$ to transmit the analog quantization error such that the desired exponent can be achieved. If this is possible, we stop there and this becomes the overall transmission scheme. Otherwise, we allocate a power level $\gamma_{i, j}$ and rate $r_{i, j}$ corresponding to the $(i, j)$ th layer as before and continue to the next layer. Note that this contains the HLS schemes of $[1,5]$ as a special case (when $N_{s}=1$ ).

2) Ordering the layers based on available power: In this variation, we allocate rate and power as in Algorithm 1 but the order of selecting the layers is not sequential. At any stage of the algorithm, we select the time layer with the maximum available power (total power minus power already allocated to superposition layers in that time layer). A new superposition layer is added to this layer with power and rate allocation as specified in Algorithm 1. Note that this is the order in which the successive refinement information from the source coder is filled and therefore the decoder should decode the layers in this order.

\section{Extensions to Multiple Block Fading Channels}

In this section we extend the results derived for the MIMO channel to the $L$-block fading MIMO channel. We assume that $K$ source samples are transmitted over $L$ blocks of length $T / L$ each. The fading coefficient for the different blocks are independent.

Lemma 3.1: If the multiplexing gain in the $i$ th layer of the broadcast scheme is $r_{i}=\frac{k L+a}{L}\left(\gamma_{i-1}-\gamma_{i}\right)+\delta$ where $k \in\{0,1, \ldots, m-1\}, a \in\{0,1, \ldots, L-1\}$ and $0 \leq \delta<\frac{\gamma_{i-1}-\gamma_{i}}{L}$, then, the achievable diversity in the $i$ th layer of the broadcast scheme, assuming that the message transmitted in the previous layers is available at the receiver, is given by

$$
d^{\star}\left(r_{i}, \gamma_{i-1}, \gamma_{i}\right)=L(m-k)(n-k) \gamma_{i-1}-(m+n-1-2 k)\left(a \gamma_{i-1}+L \delta\right) .
$$

That is, if $X=\frac{1}{\sqrt{\rho}}\left(\sum_{i=1}^{N_{s}} \sqrt{\rho^{\gamma_{i-1}}-\rho^{\gamma_{i}}} X_{i}+\sqrt{\rho^{\gamma_{s}}} N_{1}\right)$, where $X_{i}, N_{1} \sim \mathcal{N}\left(0, I^{M \times M}\right), Y_{l}=\sqrt{\frac{\rho}{M}} \mathbf{H}_{\mathbf{l}} X+N$ for $l=1, \ldots, L$, and $\mathcal{A}_{i}=\left\{H_{1}, \ldots, H_{L}: \frac{1}{L} \sum_{l=1}^{L} I\left(X_{i} ; Y_{l} \mid \mathbf{H}_{\mathbf{1}}=H_{1}, \ldots, \mathbf{H}_{\mathbf{L}}=H_{L}, X_{1}, \ldots X_{i-1}\right)<\right.$ $\left.r_{i} \log \rho\right)$ denotes the outage event set then $P\left(\mathcal{A}_{i}\right) \doteq \rho^{-d^{\star}\left(r_{i}, \gamma_{i-1}, \gamma_{i}\right)}$.

Proof:

$$
P\left(\mathcal{A}_{i}\right)=P\left(\frac{1}{L} \sum_{l=1}^{L} \log \frac{\operatorname{det}\left(I+\frac{1}{M} \rho^{\gamma_{i-1}} \mathbf{H}_{\mathbf{l}} \mathbf{H}_{\mathbf{l}}{ }^{H}\right)}{\operatorname{det}\left(I+\frac{1}{M} \rho^{\gamma_{i}} \mathbf{H}_{\mathbf{l}} \mathbf{H}_{\mathbf{l}}{ }^{H}\right)}<r_{i} \log \rho\right) .
$$

Let $\lambda_{1, l}, \ldots, \lambda_{m, l}$ denote the non-zero ordered eigenvalues of $\mathbf{H}_{\mathbf{l}} \mathbf{H}_{\mathbf{l}}{ }^{H}$ ) with $\lambda_{1, l} \leq \lambda_{2, l} \leq \ldots \lambda_{m, l}$. As in [4], let $\alpha_{j, l}=-\frac{\log \lambda_{j, l}}{\log \rho}$. Therefore, $\alpha_{1, l} \geq \alpha_{2, l} \geq \ldots \alpha_{m, l}$. At high $\operatorname{SNR}, P\left(\mathcal{A}_{i}\right) \doteq P\left(\mathcal{A}^{\prime}\right)$ where

$$
\mathcal{A}^{\prime}=\left\{\alpha: \frac{1}{L} \sum_{l=1}^{L} \sum_{j=1}^{m}\left(\left(\gamma_{i-1}-\alpha_{j, l}\right)^{+}-\left(\gamma_{i}-\alpha_{j, l}\right)^{+}\right)<r_{i}\right\} \text {. }
$$


Following in the footsteps of [4], we observe that the outage probability is given by $P\left(\mathcal{A}_{i}\right) \doteq \rho^{-d^{\star}\left(r_{i}, \gamma_{i-1}, \gamma_{i}\right)}$ where

$$
d^{\star}\left(r_{i}, \gamma_{i-1}, \gamma_{i}\right)=\inf _{\mathcal{A}^{\prime} \cap \boldsymbol{\alpha}^{+}} \sum_{j=1}^{m} \sum_{l=1}^{L}(2 j-1+n-m) \alpha_{j, l} .
$$

For $r_{i}=\frac{k L+a}{L}\left(\gamma_{i-1}-\gamma_{i}\right)+\delta$ where $k \in[0,1, \ldots, m-1], a \in[0,1, \ldots, L-1]$, and $0 \leq \delta<\frac{\gamma_{i-1}-\gamma_{i}}{L}$, the infimum in (51) occurs when $\alpha=\alpha^{*}$ where

$$
\alpha_{j, l}^{*}= \begin{cases}\gamma_{i-1}, & 1 \leq j<m-k ; \\ \gamma_{i-1}, & j=m-k, 1 \leq l<L-a \\ \gamma_{i-1}-L \delta, & j=m-k, l=L-a ; \\ 0, & j=m-k, L-a<l \leq L ; \\ 0, & m-k<j \leq m\end{cases}
$$

Hence,

$$
\begin{aligned}
d^{\star} & \left(r_{i}, \gamma_{i-1}, \gamma_{i}\right) \\
& =\sum_{j=1}^{m-k-1}(2 j-1+n-m) L \gamma_{i-1}+(2(m-k)-1+n-m)\left((L-a) \gamma_{i-1}-\delta\right) \\
& =\sum_{j=1}^{m-k}(2 j-1+n-m) L \gamma_{i-1}-(2(m-k)-1+n-m)\left(a \gamma_{i-1}+L \delta\right) \\
& =L(m-k)\left(2 \frac{m-k+1}{2}-1+n-m\right) \gamma_{i-1}-(m+n-1-2 k)\left(a \gamma_{i-1}+L \delta\right) .
\end{aligned}
$$

This then gives the desired result.

Theorem 3.1: The broadcast scheme achieves a distortion SNR exponent of $\frac{k L+a+1}{L} b, k \in\{0,1, \ldots, m-$ $1\}, a \in\{0,1, \ldots, L-1\}$ for $\frac{L(M-k)(N-k)-(a+1)(M+N-1-2 k)}{k L+a+1}<\frac{b}{L}<\frac{L(M-k)(N-k)-a(M+N-1-2 k)}{k L+a+1}$ with power and rate allocation

$$
\gamma_{i}=\alpha^{i}
$$

and

$$
r_{i}=\frac{k L+a+1}{L}\left(\gamma_{i-1}-\gamma_{i}\right)-\epsilon
$$

where

$$
\alpha=1+a+\frac{b \frac{k L+a+1}{L}-L(M-k)(N-k)}{M+N-1-2 k}
$$

for arbitrarily small $\epsilon>0$.

Proof: The power and rate allocation policy can be derived in a manner similar to that in Theorem 2.1. Here we will just verify that the specified rate and power allocation policy indeed gives the specified exponent.

We first note that for $\frac{L(M-k)(N-k)-(a+1)(M+N-1-2 k)}{k L+a+1}<\frac{b}{L}<\frac{L(M-k)(N-k)-a(M+N-1-2 k)}{k L+a+1}, 0<\alpha<1$ and therefore the specified rate and power allocation is a valid assignment. 
As in Theorem 2.1 the distortion SNR exponent is given by

$$
a_{B S}=\min \left(b \sum_{j} r_{j}, a(1), \ldots, a(i), \ldots\right)
$$

where

$$
a(i)=b \sum_{j=1}^{i-1} r_{j}+d^{*}\left(r_{i}, \gamma_{i-1}, \gamma_{i}\right)
$$

We have

$$
\lim _{i \rightarrow \infty} b \sum_{j=1}^{i} r_{j}=b \frac{k L+a+1}{L}\left(1-\lim _{i \rightarrow \infty} \gamma_{i}\right)=b \frac{k L+a+1}{L} .
$$

Furthermore, from (57) and lemma 3.1 we have

$$
\begin{aligned}
a(i)= & b \frac{k L+a+1}{L}\left(1-\gamma_{i-1}\right)+L(m-k)(n-k) \gamma_{i-1}-(m+n-1-2 k)\left((a+1) \gamma_{i-1}-\gamma_{i}\right) \\
= & b \frac{k L+a+1}{L}+(m+n-1-2 k) \gamma_{i} \\
& \quad+\gamma_{i-1}\left(L(m-k)(n-k)-(m+n-1-2 k)(a+1)-b \frac{k L+a+1}{L}\right) \\
= & b \frac{k L+a+1}{L}+(m+n-1-2 k)\left(\gamma_{i}-\alpha \gamma_{i-1}\right)=b \frac{k L+a+1}{L} .
\end{aligned}
$$

Therefore, $a_{B S}=b \frac{k L+a+1}{L}$.

By comparing with the upper bound, we observe that the broadcast scheme achieves the optimal exponent of $m b$ for $b<\frac{n-m+1}{m}$ and $M N L$ for $b>M N L^{2}$. This has been shown earlier for the $M=N=1$ case in [2] and for the $L=1$ case in [1].

Theorem 3.2: Let $c_{k L+a}=L(m+n-1-2 k) \log \left(\frac{k L+a+1}{k L+a}\right)$ for $k \in\{0,1, \ldots, m-1\}$ and $a \in$ $\{0,1, \ldots, L-1\}$. Consider the time layering scheme with a broadcast layer of bandwidth $b_{k L+a}=$ $\frac{(m-k)(n-k) L^{2}-a L(n+m-1-2 k)}{k L+a+1}$ at the end. Let $k_{1}$ and $a_{1}$ be such that $\sum_{j=k_{1} L+a_{1}+1}^{k L+a-1} c_{j}<b-b_{k L+a}<$ $\sum_{j=k_{1} L+a_{1}}^{k L+a-1} c_{j}$ where $k_{1} \in\{0,1, \ldots, m-1\}$ and $a_{1} \in\{0,1, \ldots, L-1\}$. The distortion SNR exponent is then given by

$$
a_{k L+a}(b)=\left(m-k_{1}\right)\left(n-k_{1}\right) L-\left(r_{1}-k_{1}\right) L\left(n+m-1-2 k_{1}\right)
$$

where

$$
r_{1}=\left(k_{1}+\frac{a_{1}+1}{L}\right) e^{-\frac{b-b_{k L+a}-\sum_{i=k_{1} L+a_{1}+1}^{k L+a} c_{i}}{L\left(m+n-1-2 k_{1}\right)}} .
$$

Proof: The proof is similar to that of Theorem 2.4 and is skipped here.

\section{NumERicAl RESUltS}

The achievable exponent using the proposed hybrid layering scheme along with that achievable by the HLS and broadcast schemes of [1] are shown in Fig. 7 and Fig. 8, Note that the proposed schemes outperforms the schemes in [1] for all $b$, making this the best known achievable distortion SNR exponent.

The optimal exponent can be obtained for all $b<(n-m+1) / m$ using the purely digital scheme in Section II-A. This is the first time a scheme has been shown to obtain the optimal exponent for $1 / m<b<(n-m+1) / m$. Since the scheme in Section $\Pi-\mathrm{A}$ is a special case of the scheme in Section II-C, the optimal exponent is achieved in this region by the scheme in Section $\amalg I-C$ as well.

A plot of the distortion SNR exponent for $M=N=L=2$ is shown in Fig. 10. 


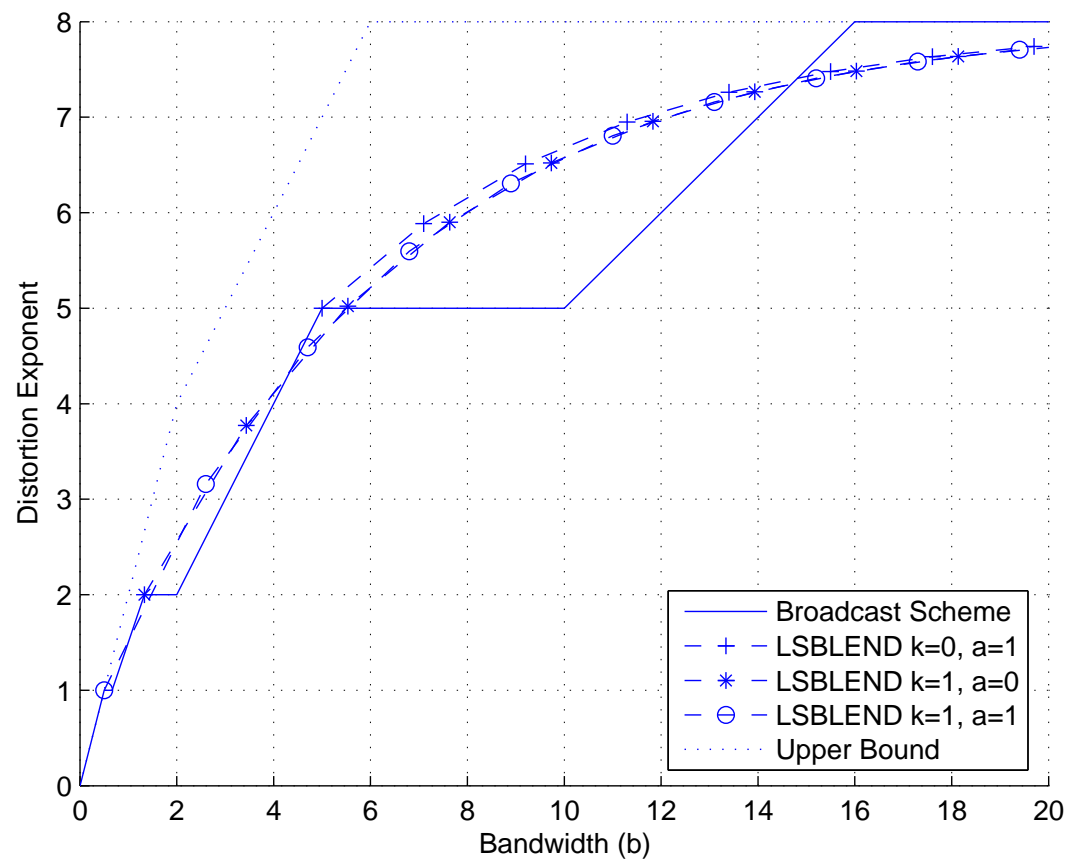

Fig. 7. Achievable distortion SNR exponent for $M=N=2$

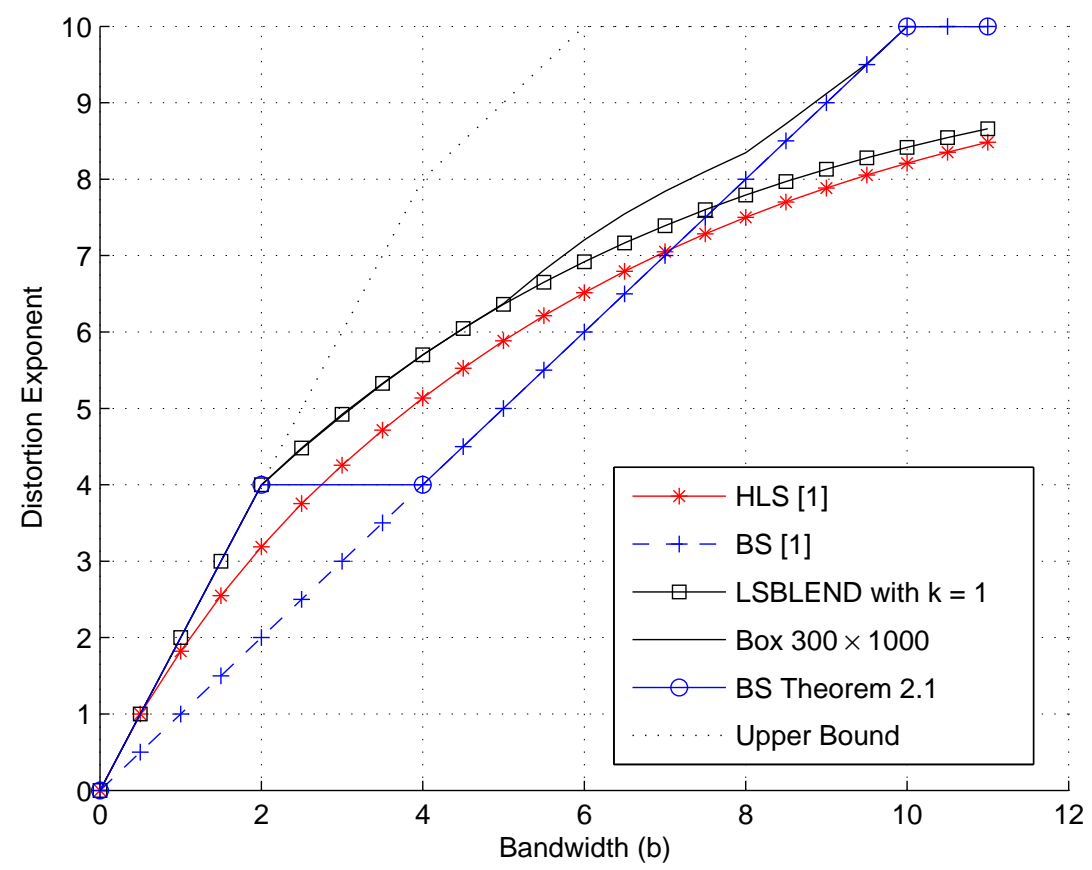

Fig. 8. Achievable distortion SNR exponent for $M=2, N=5$ 


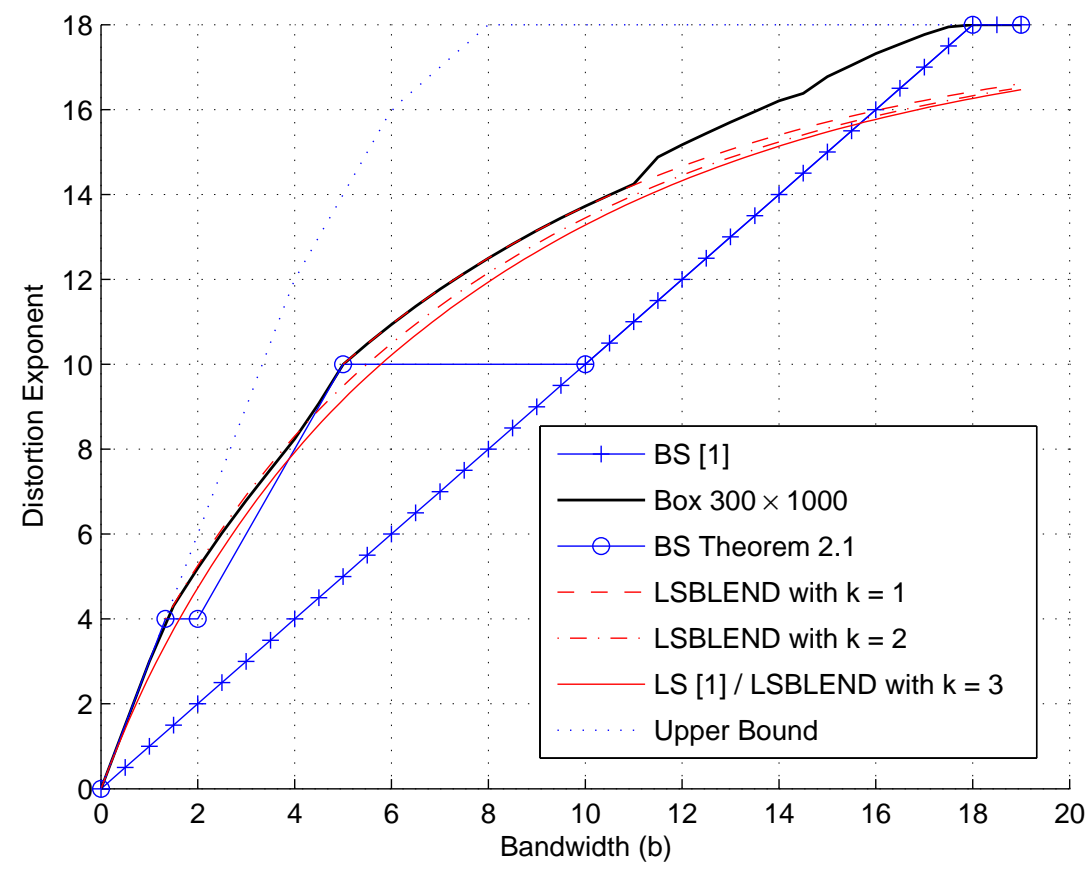

Fig. 9. Achievable distortion SNR exponent for $M=3, N=6$

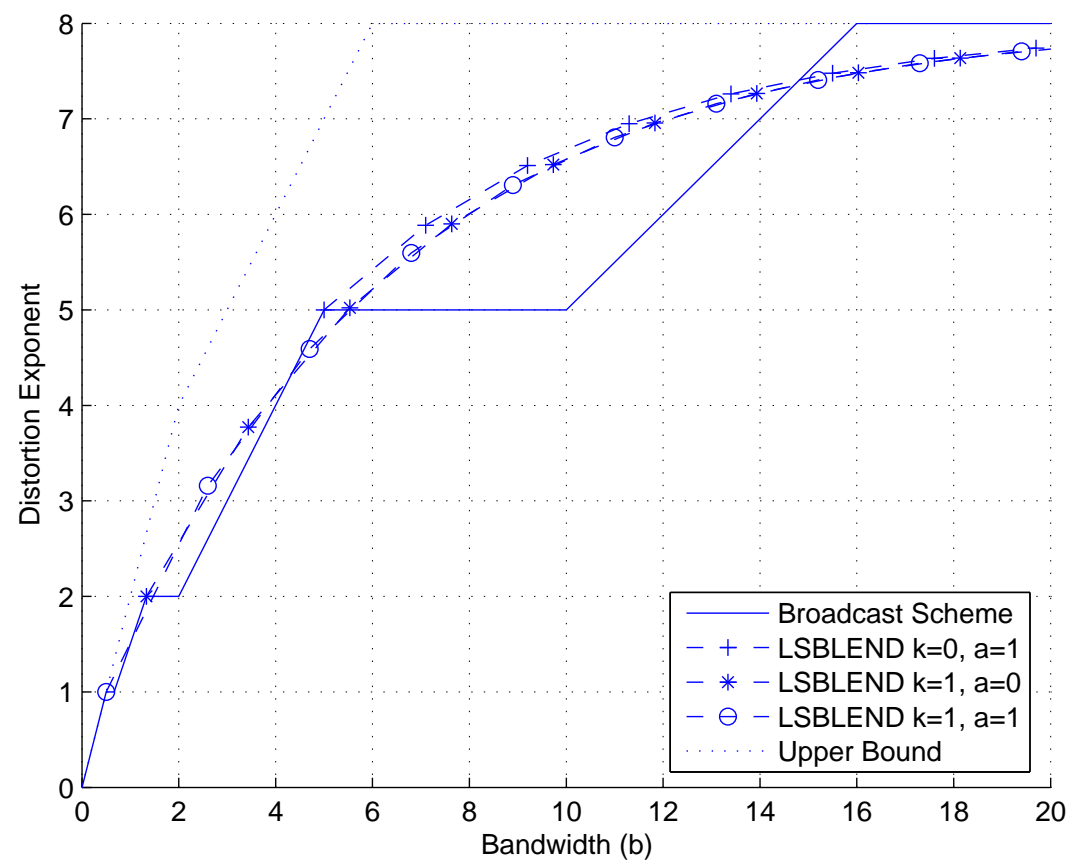

Fig. 10. Achievable distortion SNR exponent for $M=N=L=2$ 


\section{CONCLUSION}

We have proposed layering schemes for transmitting a discrete time analog source over a block fading MIMO channel. Achievable distortion SNR exponent using carefully selected rate and power allocation policies for these scheme have been studied. The achievable distortion SNR exponent obtained using these schemes is better than those reported in $[1,5]$ making this the best known distortion SNR exponent so far. Particularly, the optimal exponent is obtained for $b<(n-m+1) / m$ and $b>m n L^{2}$. We believe this is a new and surprising result. Our current research focusses on optimizing the rate and power allocation of these schemes.

\section{APPENDIX}

\section{A. Optimality of equating exponents for the BS}

The exponent $a(b)$ is given by the following optimization problem

$$
\begin{aligned}
& a(b)=-\min _{a, \gamma_{1}, \ldots, \gamma_{N_{s}}}-a \\
& \text { subject to: } \\
& C_{i}=a-b(k+1)\left(1-\gamma_{i-1}\right)-(m-k)(n-k) \gamma_{i-1}+(m+n-1-2 k)\left(\gamma_{i-1}-\gamma_{i}\right) \leq 0 \\
& \text { for } i=1,2, \ldots, N_{s} \text {; } \\
& C_{N_{s}+1}=a-b(k+1)\left(1-\gamma_{N_{s}}\right) \leq 0 \\
& \gamma_{i+1} \leq \gamma_{i} ; \quad \gamma_{N_{s}} \geq 0 ; \quad \gamma_{0}=1
\end{aligned}
$$

We solve this optimization problem by ignoring the constraints $\gamma_{i+1} \leq \gamma_{i}$ and $\gamma_{N_{s}} \geq 0$. Any solution then is an upper bound on $a(b)$. Furthermore, if the solution satisfies the ignored constraints then the solution yields $a(b)$. Consider the function $F=-a+\sum_{i=1}^{N_{s}+1} \lambda_{i} C_{i}$. Setting $d F / d \gamma_{i}=0$ we have for $i=1$ to $N_{s}-1$

$$
\begin{array}{r}
\frac{d F}{d \gamma_{i}}=-\lambda_{i}(m+n-1-2 k)+\lambda_{i+1}(b(k+1)-(m-k)(n-k)+(m+n-1-2 k))=0 \\
\Rightarrow \lambda_{i}=\frac{b(k+1)-(m-k)(n-k)+(m+n-1-2 k)}{m+n-1-2 k} \lambda_{i+1}=\alpha \lambda_{i+1} .
\end{array}
$$

For $i=N_{s}$

$$
\frac{d F}{d \gamma_{N_{s}}}=-\lambda_{N_{s}}(m+n-1-2 k)+\lambda_{N_{s}+1} b(k+1)=0
$$

Therefore, we have

$$
\lambda_{i}=\alpha^{N_{s}-i} \frac{b(k+1)}{m+n-1-2 k} \lambda_{N_{s}+1}
$$

Setting $d F / d a=0$ we have

$$
-1+\sum \lambda_{i}=0
$$

We are interested in the region $b>(m-k-1)(n-k-1) /(k+1)$ and therefore $\alpha>0$. So all $\lambda$ 's are strictly positive. Therefore, from the KKT conditions, it follows that the optimal solution satisfies $C_{i}=0$ for $i=1$ to $N_{S}+1$. 


\section{B. Optimality of equating exponents for LSBLEND}

The exponent $a(b)$ is given by the following optimization problem

$$
\begin{aligned}
& a(b)=-\min _{a, r_{1}, \ldots, r_{N_{t}}}-a \\
& \text { subject to: } \\
& C_{i}=a-\frac{b-b_{k}}{N_{t}} \sum_{j=0}^{i-1} r_{j}-d^{*}\left(r_{i}\right) \leq 0 \quad \text { for } i=1,2, \ldots, N_{t} ; \\
& C_{N_{t}+1}=a-\frac{b-b_{k}}{N_{t}} \sum_{j=0}^{N_{t}} r_{j}+b_{k}(k+1) \leq 0 ; \\
& r_{i} \geq 0 ; \quad r_{i}<m ; \quad r_{0}=0 .
\end{aligned}
$$

As in Appendix $\AA$ we ignore the constraints $0<r_{i}<m$ and consider the function $F=-a+\sum_{i=1}^{N_{t}+1} \lambda_{i} C_{i}$. Setting $d F / d r_{i}=0$ we have for $i=1$ to $N_{t}$

$$
\frac{d F}{d r_{i}}=-\frac{b-b_{k}}{N_{t}} \sum_{j=i+1}^{N_{t}+1} \lambda_{j}-\lambda_{i} \frac{d}{d r_{i}}\left(d^{*}\left(r_{i}\right)\right)=0 .
$$

Note that $\frac{b-b_{k}}{N_{t}}>0$ and $\frac{d}{d r_{i}}\left(d^{*}\left(r_{i}\right)\right)<0$. Starting from $i=N_{t}$ and solving recursively for $\lambda_{i}$ in terms of $\lambda_{N_{t}+1}$ we observe that the $\lambda_{i}$ 's are of form $\alpha_{i} \lambda_{N_{s}+1}$ where $\alpha_{i}>0$. By setting $d F / d a=0$ we have $\sum_{i=1}^{N_{t}+1} \lambda_{i}=1$. Therefore, $\lambda_{i}>0$ for all $i$ and hence, from the KKT conditions, we conclude that the optimal solution satisfies $C_{i}=0$ for $i=1$ to $N_{t}+1$.

\section{REFERENCES}

[1] D. Gunduz and E. Erkip. Joint source channel codes for mimo block fading channels. Submitted to the IEEE Transactions on Information Theory, April 2006.

[2] D. Gunduz and E. Erkip. Distortion exponent of parallel fading channels. In ISIT, Seattle WA, USA, July 2006.

[3] D. Gunduz and E. Erkip. Source and channel coding for quasi-static fading channels. In 39th Asilomar Conf. on Sig., Syst. and Comp., Monterey CA, USA, Nov. 2004.

[4] L. Zheng and D. Tse. Diversity and multiplexing: a fundamental tradeoff in multiple-antenna channels. IEEE Transactions on Information Theory, 49(5):1073-1096, May 2003.

[5] G. Caire and K.R. Narayanan. On the SNR exponent of hybrid digital analog space time codes. In Allerton Conf. Commun. Control and Computing, Monticello, IL, USA, Oct 2005.

[6] G. Caire and K.R. Narayanan. On the distortion SNR exponent of hybrid digital-analog space time coding. submitted to IEEE Transactions on Information Theory, 2006.

[7] K.R. Narayanan and G. Caire. Further results on the SNR exponent of hybrid digital analog space time codes. In UCSD Workshop on Info. Theory and Its Applications, San Diego CA, USA, Feb 2006.

[8] D. Gunduz and E. Erkip. Distortion exponent of mimo fading channels. In ITW, Punta del Este, Uruguay, March 2006.

[9] T. Holliday and A.J. Goldsmith. Joint source and channel coding for mimo systems. In Allerton Conf. Commun. Control and Computing, pages 1302-1311, Monticello IL, USA, Oct. 2004.

[10] T. Holliday and A. Goldsmith. Optimizing end-to-end distortion in MIMO systems. In IEEE ISIT 2005, pages 1671-1675, Adelaide, Australia, September, 4-10 2005.

[11] J.N. Laneman, E. Martinian, G.W. Wornell, J.G. Apostolopoulos, and S.J. Wee. Comparing application- and physical-layer approaches to diversity on wireless channels. In Intl Conf Communications, pages 2678-2882, Alaska USA, May 2003.

[12] J.N. Laneman, E. Martinian, and G.W. Wornell. Source-channel diversity approaches for multimedia communication. In ISIT, Chicago USA, July 2004. 
[13] J.N. Laneman, E. Martinian, G.W. Wornell, and J.G. Apostolopoulos. Source channel diversity for parallel channels. IEEE Transactions on Information Theory, 51(10):3518-3539, Oct 2005.

[14] B. Dunn and J.N. Laneman. Characterizing source-channel diversity approaches beyond the distortion exponent. In Allerton Conf. Commun. Control and Computing, Monticello IL, USA, Oct 2005.

[15] K. Bhattad, K.R. Narayanan, and G. Caire. On the distortion exponent of some layered transmission schemes. In 40th Asilomar Conf. on Sig., Syst. and Comp., Monterey CA, USA, November 2006. 